\title{
Embeddings from the point of view of immersion theory : Part I
}

\author{
Michael Weiss \\ Department of Mathematics, University of Aberdeen \\ Aberdeen, AB24 3UE, UK \\ Email: m.weiss@maths.abdn.ac.uk
}

\begin{abstract}
Let $M$ and $N$ be smooth manifolds without boundary. Immersion theory suggests that an understanding of the space of smooth embeddings emb $(M, N)$ should come from an analysis of the cofunctor $V \mapsto \operatorname{emb}(V, N)$ from the poset $\mathcal{O}$ of open subsets of $M$ to spaces. We therefore abstract some of the properties of this cofunctor, and develop a suitable calculus of such cofunctors, Goodwillie style, with Taylor series and so on. The terms of the Taylor series for the cofunctor $V \mapsto \operatorname{emb}(V, N)$ are explicitly determined. In a sequel to this paper, we introduce the concept of an analytic cofunctor from $\mathcal{O}$ to spaces, and show that the Taylor series of an analytic cofunctor $F$ converges to $F$. Deep excision theorems due to Goodwillie and Goodwillie-Klein imply that the cofunctor $V \mapsto \operatorname{emb}(V, N)$ is analytic when $\operatorname{dim}(N)-\operatorname{dim}(M) \geq 3$.
\end{abstract}

AMS Classification numbers Primary: 57R40

Secondary: 57R42

Keywords: Embedding, immersion, calculus of functors

Proposed: Ralph Cohen

Seconded: Haynes Miller, Gunnar Carlsson

Received: 10 May 1998

Revised: 5 May 1999

Copyright Geometry and Topology 


\section{Introduction}

Recently Goodwillie [9], [10], [11] and Goodwillie-Klein [12] proved higher excision theorems of Blakers-Massey type for spaces of smooth embeddings. In conjunction with a calculus framework, these lead to a calculation of such spaces when the codimension is at least 3 . Here the goal is to set up the calculus framework. This is very similar to Goodwillie's calculus of homotopy functors [6], [7], [8], but it is not a special case. Much of it has been known to Goodwillie for a long time. For some history and a slow introduction, see [23]. If a reckless introduction is required, read on-but be prepared for Grothendieck topologies [18] and homotopy limits [1], [23, section 1].

Let $M$ and $N$ be smooth manifolds without boundary. Write $\operatorname{imm}(M, N)$ for the space of smooth immersions from $M$ to $N$. Let $\mathcal{O}$ be the poset of open subsets of $M$, ordered by inclusion. One of the basic ideas of immersion theory since Gromov [14], [16], [19] is that $\operatorname{imm}(M, N)$ should be regarded as just one value of the cofunctor $V \mapsto \operatorname{imm}(V, N)$ from $\mathcal{O}$ to spaces. Here $\mathcal{O}$ is treated as a category, with exactly one morphism $V \rightarrow W$ if $V \subset W$, and no morphism if $V \not \subset W$; a cofunctor is a contravariant functor.

The poset or category $\mathcal{O}$ has a Grothendieck topology [18, III.2.2] which we denote by $\mathcal{J}_{1}$. Namely, a family of morphisms $\left\{V_{i} \rightarrow W \mid i \in S\right\}$ qualifies as a covering in $\mathcal{J}_{1}$ if every point of $W$ is contained in some $V_{i}$. More generally, for each $k>0$ there is a Grothendieck topology $\mathcal{J}_{k}$ on $\mathcal{O}$ in which a family of morphisms $\left\{V_{i} \rightarrow W \mid i \in S\right\}$ qualifies as a covering if every finite subset of $W$ with at most $k$ elements is contained in some $V_{i}$. We will say that a cofunctor $F$ from $\mathcal{O}$ to spaces is a homotopy sheaf with respect to the Grothendieck topology $\mathcal{J}_{k}$ if for any covering $\left\{V_{i} \rightarrow W \mid i \in S\right\}$ in $\mathcal{J}_{k}$ the canonical map

$$
F(W) \longrightarrow \operatorname{holim}_{\emptyset \neq R \subset S} F\left(\cap_{i \in R} V_{i}\right)
$$

is a homotopy equivalence. Here $R$ runs through the finite nonempty subsets of $S$. In view of the homotopy invariance properties of homotopy inverse limits, the condition means that the values of $F$ on large open sets are sufficiently determined for the homotopy theorist by the behavior of $F$ on certain small open sets; however, it depends on $k$ how much smallness we can afford.- The main theorem of immersion theory is that the cofunctor $V \mapsto \operatorname{imm}(V, N)$ from $\mathcal{O}$ to spaces is a homotopy sheaf with respect to $\mathcal{J}_{1}$, provided $\operatorname{dim}(N)$ is greater than $\operatorname{dim}(M)$ or $\operatorname{dim}(M)=\operatorname{dim}(N)$ and $M$ has no compact component.

In this form, the theorem may not be very recognizable. It can be decoded as follows. Let $Z$ be the space of all triples $(x, y, f)$ where $x \in M, y \in N$ and $f: T_{x} M \rightarrow T_{y} N$ is a linear monomorphism. Let $p: Z \rightarrow M$ be the projection 
to the first coordinate. For $V \in \mathcal{O}$ we denote by $\Gamma(p ; V)$ the space of partial sections of $p$ defined over $V$. It is not hard to see that $V \mapsto \Gamma(p ; V)$ is a homotopy sheaf with respect to $\mathcal{J}_{1}$. (Briefly: if $\left\{V_{i} \rightarrow W\right\}$ is a covering in $\mathcal{J}_{1}$, then the canonical map $q$ : hocolim $\cap_{R} \cap_{i \in R} V_{i} \rightarrow W$ is a homotopy equivalence according to [24], so that $\Gamma(p ; W) \simeq \Gamma\left(q^{*} p\right) \cong \operatorname{holim}_{R} \Gamma\left(p ; \cap_{i \in R} V_{i}\right)$. ) There is an obvious inclusion

$$
\operatorname{imm}(V, N) \hookrightarrow \Gamma(p ; V)
$$

which we regard as a natural transformation between cofunctors in the variable $V$. We want to show that $(*)$ is a homotopy equivalence for every $V$, in particular for $V=M$; this is the decoded version of the main theorem of immersion theory, as stated in Haefliger-Poenaru [15] for example (in the PL setting). By inspection, $(*)$ is indeed a homotopy equivalence when $V$ is diffeomorphic to $\mathbb{R}^{m}$. An arbitrary $V$ has a smooth triangulation and can then be covered by the open stars $V_{i}$ of the triangulation. Since $(*)$ is a homotopy equivalence for the $V_{i}$ and their finite intersections, it is a homotopy equivalence for $V$ by the homotopy sheaf property.

Let us now take a look at the space of smooth embeddings $\operatorname{emb}(M, N)$ from the same point of view. As before, we think of $\operatorname{emb}(M, N)$ as just one value of the cofunctor $V \mapsto \operatorname{emb}(V, N)$ from $\mathcal{O}$ to spaces. The cofunctor is clearly not a homotopy sheaf with respect to the Grothendieck topology $\mathcal{J}_{1}$, except in some very trivial cases. For if it were, the inclusion

$$
\operatorname{emb}(V, N) \stackrel{\subset}{\longrightarrow} \operatorname{imm}(V, N)
$$

would have to be a homotopy equivalence for every $V \in \mathcal{O}$, since it is clearly a homotopy equivalence when $V$ is diffeomorphic to $\mathbb{R}^{m}$. In fact it is quite appropriate to think of the cofunctor $V \mapsto \operatorname{imm}(V, N)$ as the homotopy sheafification of $V \mapsto \operatorname{emb}(V, N)$, again with respect to $\mathcal{J}_{1}$. The natural transformation (**) has a suitable universal property which justifies the terminology.

Clearly now is the time to try out the smaller Grothendieck topologies $\mathcal{J}_{k}$ on $\mathcal{O}$. For each $k>0$ the cofunctor $V \mapsto \operatorname{emb}(V, N)$ has a homotopy sheafification with respect to $\mathcal{J}_{k}$. Denote this by $V \mapsto T_{k} \operatorname{emb}(V, N)$. Thus $V \mapsto T_{k} \operatorname{emb}(V, N)$ is a homotopy sheaf on $\mathcal{O}$ with respect to $\mathcal{J}_{k}$ and there is a natural transformation

$(* * *) \quad \operatorname{emb}(V, N) \longrightarrow T_{k} \operatorname{emb}(V, N)$

which should be regarded as the best approximation of $V \mapsto \operatorname{emb}(V, N)$ by a cofunctor which is a homotopy sheaf with respect to $\mathcal{J}_{k}$. I do not know of any convincing geometric interpretations of $T_{k} \operatorname{emb}(V, N)$ except of course in the case $k=1$, which we have already discussed. As Goodwillie explained to me, 
his excision theorem for diffeomorphisms [9], [10], [11] and improvements due to Goodwillie-Klein [12] imply that $(* * *)$ is $(k(n-m-2)+1-m)$-connected where $m=\operatorname{dim}(M)$ and $n=\operatorname{dim}(N)$. In particular, if the codimension $n-m$ is greater than 2 , then the connectivity of $(* * *)$ tends to infinity with $k$. The suggested interpretation of this result is that, if $n-m>2$, then $V \mapsto \operatorname{emb}(V, N)$ behaves more and more like a homotopy sheaf on $\mathcal{O}$, with respect to $\mathcal{J}_{k}$, as $k$ tends to infinity.

Suppose now that $M \subset N$, so that $\operatorname{emb}(V, N)$ is a based space for each open $V \subset M$. Then the following general method for calculating or partially calculating $\operatorname{emb}(M, N)$ is second to none. Try to determine the cofunctors

$$
V \mapsto \text { homotopy fiber of }\left[T_{k} \operatorname{emb}(V, N) \rightarrow T_{k-1} \operatorname{emb}(V, N)\right]
$$

for the first few $k>0$. These cofunctors admit a surprisingly simple description in terms of configuration spaces; see Theorem 8.5, and [23]. Try to determine the extensions (this tends to be very hard) and finally specialize, letting $V=M$. This program is already outlined in Goodwillie's expanded thesis [9, section Intro.C] for spaces of concordance embeddings (a special case of a relative case), with a pessimistic note added in revision: “ . . it might never be [written up] ...". It is also carried out to some extent in a simple case in [23]. More details on the same case can be found in Goodwillie-Weiss [13].

Organization Part I (this paper) is about the series of cofunctors $V \mapsto T_{k} \operatorname{emb}(V, N)$, called the Taylor series of the cofunctor $V \mapsto \operatorname{emb}(V, N)$. It is also about Taylor series of other cofunctors of a similar type, but it does not address convergence questions. These will be the subject of Part II ([13], joint work with Goodwillie).

Convention Since homotopy limits are so ubiquitous in this paper, we need a "convenient" category of topological spaces with good homotopy limits. The category of fibrant simplicial sets is such a category. In the sequel, "Space" with a capital S means fibrant simplicial set. As a rule, we work with (co-)functors whose values are Spaces and whose arguments are spaces (say, manifolds). However, there are some situations, for example in section 9, where it is a good idea to replace the manifolds by their singular simplicial sets. Such a replacement is often understood. 


\section{Good Cofunctors}

1.1 Definition A smooth codimension zero embedding $i_{1}: V \rightarrow W$ between smooth manifolds without boundary is an isotopy equivalence if there exists a smooth embedding $i_{2}: W \rightarrow V$ such that $i_{1} i_{2}$ and $i_{2} i_{1}$ are smoothly isotopic to $\mathrm{id}_{W}$ and $\mathrm{id}_{V}$, respectively.

In the sequel $M$ is a smooth manifold without boundary, and $\mathcal{O}$ is the poset of open subsets of $M$, ordered by inclusion. Usually we think of $\mathcal{O}$ as a category, with exactly one morphism $V \rightarrow W$ if $V \subset W$, and no morphism if $V \not \subset W$. A cofunctor (=contravariant functor) $F$ from $\mathcal{O}$ to Spaces is good if it satisfies the following conditions.

(a) $F$ takes isotopy equivalences to homotopy equivalences.

(b) For any sequence $\left\{V_{i} \mid i \geq 0\right\}$ of objects in $\mathcal{O}$ with $V_{i} \subset V_{i+1}$ for all $i \geq 0$, the following canonical map is a weak homotopy equivalence:

$$
F\left(\cup_{i} V_{i}\right) \longrightarrow \operatorname{holim}_{i} F\left(V_{i}\right) .
$$

1.2 Notation $\mathcal{F}$ is the category of all good cofunctors from $\mathcal{O}$ to Spaces. The morphisms in $\mathcal{F}$ are the natural transformations. A morphism $g: F_{1} \rightarrow F_{2}$ is an equivalence if $g_{V}: F_{1}(V) \longrightarrow F_{2}(V)$ is a homotopy equivalence for all $V$ in $\mathcal{O}$. Two objects in $\mathcal{F}$ are equivalent if they can be related by a chain of equivalences.

1.3 Examples For any smooth manifold $N$ without boundary, there are cofunctors from $\mathcal{O}$ to Spaces given by $V \mapsto \operatorname{emb}(V, N)$ (Space of smooth embeddings) and $V \mapsto \operatorname{imm}(V, N)$ (Space of smooth immersions). To be more precise, we think of $\operatorname{emb}(V, N)$ and $\operatorname{imm}(V, N)$ as geometric realizations of simplicial sets: for example, a 0 -simplex of $\operatorname{imm}(V, N)$ is a smooth immersion $V \rightarrow N$, and a 1-simplex in $\operatorname{imm}(V, N)$ is a smooth immersion $V \times \Delta^{1} \rightarrow N \times \Delta^{1}$ respecting the projection to $\Delta^{1}$.

1.4 Proposition The cofunctors $\operatorname{imm}(-, N)$ and $\operatorname{emb}(-, N)$ are good. 
Part (a) of goodness is easily verified for both $\operatorname{imm}(-, N)$ and $\operatorname{emb}(-, N)$. Namely, suppose that $i_{1}: V \rightarrow W$ is an isotopy equivalence between smooth manifolds, with isotopy inverse $i_{2}: W \rightarrow V$ and isotopies $\left\{h_{t}: V \rightarrow V\right\},\left\{k_{t}: W\right.$ $\rightarrow W\}$ from $i_{2} i_{1}$ to $\operatorname{id}_{V}$ and from $i_{1} i_{2}$ to id $W$, respectively. Then $\left\{h_{t}: V \rightarrow V\right\}$ gives rise to a map of simplicial sets

$$
\operatorname{imm}(V, N) \times \Delta^{1} \rightarrow \operatorname{imm}(V, N)
$$

which is a homotopy from $\left(i_{2} i_{1}\right)^{*}$ to the identity. Similarly $\left\{k_{t}: W \rightarrow W\right\}$ gives rise to a homotopy connecting $\left(i_{1} i_{2}\right)^{*}$ and the identity on $\operatorname{imm}(W, N)$. Therefore $\operatorname{imm}(-, N)$ is isotopy invariant. The same reasoning applies to $\operatorname{emb}(-, N)$.

To establish part (b) of goodness, we note that it is enough to consider the case where $M$ is connected. Then a sequence $\left\{V_{i}\right\}$ as in part (b) will either be stationary, in which case we are done, or almost all the $V_{i}$ are open manifolds (no compact components).

1.5 Lemma Suppose that $V \in \mathcal{O}$ has no compact components. Suppose also that $V=\cup_{i} K_{i}$ where each $K_{i}$ is a smooth compact manifold with boundary, contained in the interior of $K_{i+1}$, for $i \geq 0$. Then the canonical maps

$$
\operatorname{imm}(V, N) \rightarrow \underset{i}{\operatorname{holim} \operatorname{imm}}\left(K_{i}, N\right), \quad \operatorname{emb}(V, N) \rightarrow \underset{i}{\operatorname{holim} \operatorname{emb}}\left(K_{i}, N\right)
$$

are homotopy equivalences.

Proof By the isotopy extension theorem, the restriction from $\operatorname{emb}\left(K_{i+1}, N\right)$ to $\operatorname{emb}\left(K_{i}, N\right)$ is a Kan fibration of simplicial sets. It is a standard result of immersion theory, much more difficult to establish than the isotopy extension theorem, that the restriction map from $\operatorname{imm}\left(K_{i+1}, N\right)$ to $\operatorname{imm}\left(K_{i}, N\right)$ is a Kan fibration. See especially Haefliger-Poenaru [15]; although this is written in PL language, it is one of the clearest references.

Let $\operatorname{emb}_{!}(V, N)$ be the Space of thick embeddings $V \rightarrow N$, that is, embeddings $f: V \rightarrow N$ together with a sober extension of $f$ to an embedding $D\left(\nu_{f}\right) \rightarrow N$, where $D\left(\nu_{f}\right)$ is the total space of the normal disk bundle of $f$. (The word sober means that the resulting bundle isomorphism between the normal bundle of $M$ in $D\left(\nu_{f}\right)$ and $\nu_{f}$ itself is the canonical one.) Define emb! $\left(K_{i}, N\right)$ similarly. In the commutative diagram

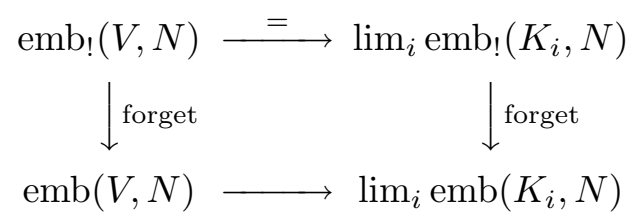

Geometry and Topology, Volume 3 (1999) 
the left-hand vertical arrow is a homotopy equivalence by inspection, and the right-hand vertical arrow is a homotopy equivalence because, according to Bousfield-Kan [1], the canonical map from the limit to the homotopy limit of a tower of Kan fibrations is a homotopy equivalence of simplicial sets. (Hence the limits in the right-hand column could be replaced by homotopy limits.) Hence the lower horizontal arrow is a homotopy equivalence. Note: the lower horizontal arrow is not always an isomorphism of simplicial sets-injective immersions are not always embeddings.

Suppose now that $V=\cup_{i} V_{i}$ as in part (b) of goodness, and that $V$ has no compact components. Each $V_{i}$ can be written as a union $\cup_{j} K_{i j}$ where each $K_{i j}$ is smooth compact with boundary, and $K_{i j}$ is contained in the interior of $K_{i(j+1)}$. Moreover we can arrange that $K_{i j}$ is also contained in the interior of $K_{(i+1) j}$. Writing $F(-)$ to mean $\operatorname{imm}(-, N)$, we have a commutative diagram of restriction maps

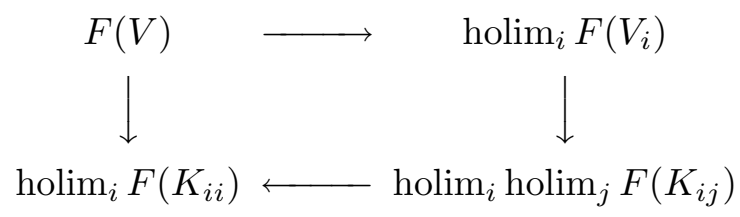

where the vertical arrows are homotopy equivalences by 1.5 and the lower horizontal arrow is a homotopy equivalence by $[4,9.3]$. (Here we identify $\operatorname{holim}_{i} \operatorname{holim}_{j}$ with $\left.\operatorname{holim}_{i j}.\right)$ This shows that the cofunctor $\operatorname{imm}(-, N)$ has property (b). The same argument applies to the cofunctor emb $(-, N)$. Hence 1.4 is proved.

\section{Polynomial Cofunctors}

The following, up to and including Definiton 2.2, is a quotation from [23]. Suppose that $F$ belongs to $\mathcal{F}$ and that $V$ belongs to $\mathcal{O}$, and let $A_{0}, A_{1}, \ldots, A_{k}$ be pairwise disjoint closed subsets of $V$. Let $\mathcal{P}_{k+1}$ be the power set of $[k]=$ $\{0,1, \ldots, k\}$. This is a poset, ordered by inclusion. We make a functor from $\mathcal{P}_{k+1}$ to Spaces by

$$
S \mapsto F\left(V \backslash \cup_{i \in S} A_{i}\right)
$$

for $S$ in $\mathcal{P}_{k+1}$. Recall that, in general, a functor $\mathcal{X}$ from $\mathcal{P}_{k+1}$ to Spaces is called a $(k+1)$-cube of Spaces. 
2.1 Definition ([6], [7]) The total homotopy fiber of the cube $\mathcal{X}$ is the homotopy fiber of the canonical map

$$
\mathcal{X}(\emptyset) \longrightarrow \operatorname{holim}_{S \neq \emptyset} \mathcal{X}(S) .
$$

If the canonical map $\mathcal{X}(\emptyset) \rightarrow \operatorname{holim}_{S \neq \emptyset} \mathcal{X}(S)$ is a homotopy equivalence, then $\mathcal{X}$ is homotopy Cartesian or just Cartesian.

A cofunctor $\mathcal{Y}$ from $\mathcal{P}_{k+1}$ to spaces will also be called a cube of spaces, since $\mathcal{P}_{k+1}$ is isomorphic to its own opposite. The total homotopy fiber of $\mathcal{Y}$ is the homotopy fiber of $\mathcal{Y}([k]) \rightarrow \operatorname{holim}_{S \neq[k]} \mathcal{Y}(S)$.

Inspired by $[7,3.1]$ we decree:

2.2 Definition The cofunctor $F$ is polynomial of degree $\leq k$ if the $(k+1)$ cube $(*)$ is Cartesian for arbitrary $V$ in $\mathcal{O}$ and pairwise disjoint closed subsets $A_{0}, \ldots, A_{k}$ of $V$.

Remark In Goodwillie's calculus of functors, a functor from spaces to spaces is of degree $\leq k$ if it takes strongly cocartesian $(k+1)$-cubes to Cartesian $(k+1)$-cubes. The pairwise disjointness condition in 2.2 is there precisely to ensure that the cube given by $S \mapsto V \backslash \cup_{i \in S} A_{i}$ is strongly cocartesian.

2.3 Example The cofunctor $V \mapsto \operatorname{imm}(V, N)$ is polynomial of degree $\leq 1$ if either $\operatorname{dim}(N)>\operatorname{dim}(M)$ or the dimensions are equal and $M$ has no compact component. This amounts to saying that for open subsets $V_{1}$ and $V_{2}$ of $M$, the following square of restriction maps is a homotopy pullback square:

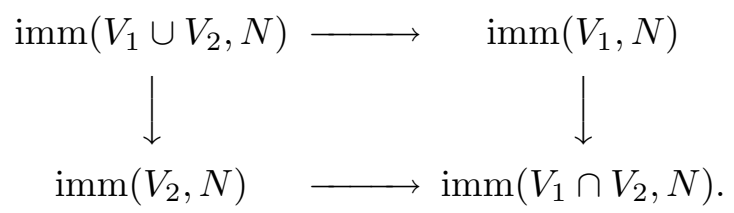

To prove this we use lemma 1.5. Accordingly it is enough to prove that

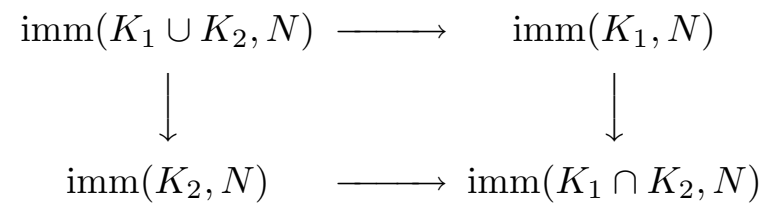

is a homotopy pullback square whenever $K_{1}, K_{2} \subset M$ are smooth compact codimension zero submanifolds of $M$ whose boundaries intersect transversely. (Then $K_{1} \cap K_{2}$ is smooth "with corners".) But (**) is a strict pullback square of Spaces in which all arrows are (Kan) fibrations, by [15]. 
2.4 Example Fix a space $X$, and for $V \in \mathcal{O}$ let $\left(\begin{array}{l}V \\ k\end{array}\right)$ be the configuration space of unordered $k$-tuples in $V$. This is the complement of the fat diagonal in the $k$-fold symmetric product $(V \times V \times \ldots \times V) / \Sigma_{k}$. The cofunctor

$$
V \mapsto \operatorname{map}\left(\left(\begin{array}{l}
V \\
k
\end{array}\right), X\right)
$$

where map denotes a simplicial set of maps, is polynomial of degree $\leq k$. Here is a sketch proof: Let $A_{0}, A_{1}, \ldots, A_{k}$ be pairwise disjoint closed subsets of $V$. Any unordered $k$-tuple in $V$ must have empty intersection with one of the $A_{i}$. Therefore

$$
\left(\begin{array}{l}
V \\
k
\end{array}\right)=\bigcup_{i}\left(\begin{array}{c}
V \backslash A_{i} \\
k
\end{array}\right)
$$

and it is not hard to deduce that the canonical map

$$
\underset{\substack{S \subset\{0,1, \ldots, k\} \\
S \neq \emptyset}}{\operatorname{hocolim}}\left(\begin{array}{c}
V \backslash \cup_{i \in S} A_{i} \\
k
\end{array}\right) \longrightarrow\left(\begin{array}{l}
V \\
k
\end{array}\right)
$$

is a homotopy equivalence. Compare [24]. Applying map $(-, X)$ turns the homotopy colimit into a homotopy limit and the proof is complete.

2.5 Example Let $\mathcal{A}$ be a small category and let $\phi: \mathcal{A} \rightarrow \mathcal{F}$ be a functor, which we will write in the form $a \mapsto \phi_{a}$. Suppose that each $\phi_{a}$ is polynomial of degree $\leq k$. Then

$$
V \mapsto \operatorname{holim}_{a} \phi_{a}(V)
$$

is in $\mathcal{F}$, and is polynomial of degree $\leq k$. Special case: For $\mathcal{A}$ take the poset of nonempty subsets of $\{0,1\}$, and conclude that $\mathcal{F}$ is closed under homotopy pullbacks.

\section{Special Open Sets}

Let $\mathcal{O} k$ consist of all open subsets of $M$ which are diffeomorphic to a disjoint union of at most $k$ copies of $\mathbb{R}^{m}$, where $m=\operatorname{dim}(M)$. We think of $\mathcal{O} k$ as a full subcategory of $\mathcal{O}$. There is an important relationship between $\mathcal{O} k$ and definition 2.2 which we will work out later, and which is roughly as follows. A good cofunctor from $\mathcal{O}$ to Spaces which is polynomial of degree $\leq k$ is determined by its restriction to $\mathcal{O} k$, and moreover the restriction to $\mathcal{O} k$ can be arbitrarily prescribed.- In this section, however, we merely examine the homotopy type of $|\mathcal{O} k|$ and use the results to study the process of inflation (right Kan extension) of a cofunctor along the inclusion $\mathcal{O} k \hookrightarrow \mathcal{O}$. 
For the proof of lemma 3.9 below, we need double categories [17]. Recall first that a category $\mathcal{C}$ consists of two classes, $o b(\mathcal{C})$ and $\operatorname{mor}(\mathcal{C})$, as well as maps $s, t: \operatorname{mor}(\mathcal{C}) \rightarrow o b(\mathcal{C})$ (source and target) and $1: o b(\mathcal{C}) \rightarrow \operatorname{mor}(\mathcal{C})$ and

$$
\circ: \operatorname{mor}(\mathcal{C})_{t} \times{ }_{s} \operatorname{mor}(\mathcal{C}) \longrightarrow \operatorname{mor}(\mathcal{C})
$$

(composition), where ${ }_{t} \times_{s}$ denotes the fibered product (or pullback) over $o b(\mathcal{C})$. The maps $s, t, 1$ and $\circ$ satisfy certain relations. A double category is a category object in the category of categories. Thus a double category $\mathcal{C}$ consists of two categories, $o b(\mathcal{C})$ and $\operatorname{mor}(\mathcal{C})$, as well as functors $s, t: \operatorname{mor}(\mathcal{C}) \rightarrow o b(\mathcal{C})$ (source and target) and 1: ob(C) $\rightarrow \operatorname{mor}(\mathcal{C})$ and

$$
\circ: \operatorname{mor}(\mathcal{C})_{t} \times{ }_{s} \operatorname{mor}(\mathcal{C}) \longrightarrow \operatorname{mor}(\mathcal{C})
$$

(composition) where ${ }_{t} \times_{s}$ denotes the fibered product (or pullback) over $o b(\mathcal{C})$. These functors $s, t, 1$ and $\circ$ satisfy the expected relations. Alternative definition: A double category consists of four classes, $\operatorname{ob}(o b(\mathcal{C})), \operatorname{mor}(o b(\mathcal{C}))$, $o b(\operatorname{mor}(\mathcal{C}))$ and $\operatorname{mor}(\operatorname{mor}(\mathcal{C}))$, and certain maps relating them ... This definition has the advantage of being more symmetric. In particular, we see that a double category $\mathcal{C}$ determines two ordinary categories, the horizontal category $\mathcal{C}_{h}$ and the vertical category $\mathcal{C}_{v}$, both with object class $o b(o b(\mathcal{C}))$. The morphism class of $\mathcal{C}_{h}$ is $\operatorname{ob}(\operatorname{mor}(\mathcal{C}))$, that of $\mathcal{C}_{v}$ is $\operatorname{mor}(o b(\mathcal{C}))$.

The nerve of a double category $\mathcal{C}$ is a bisimplicial set, denoted by $|\mathcal{C}|$.

3.1 Example Suppose that two groups $H$ and $V$ act on the same set $S$ (both on the left). Make a double category $\mathcal{C}$ with $o b(o b(\mathcal{C}))=S, o b(\operatorname{mor}(\mathcal{C}))=S \times H$, $\operatorname{mor}(o b(\mathcal{C}))=S \times V$, and

$$
\operatorname{mor}(\operatorname{mor}(\mathcal{C})):=\left\{\left(s, h_{1}, h_{2}, v_{1}, v_{2}\right) \mid v_{2} h_{1} s=h_{2} v_{1} s\right\} .
$$

Thus an element in $\operatorname{mor}(\operatorname{mor}(\mathcal{C}))$ is a "commutative diagram"

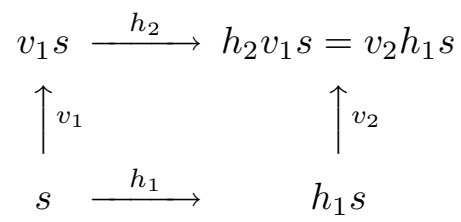

where the vertices are in $S$ and the labelled arrows indicate left multiplication by suitable elements of $H$ or $V$.

3.2 Example An ordinary category $\mathcal{A}$ gives rise to a double category denoted $\mathcal{A} \mathcal{A}$ with $(\mathcal{A A})_{h}=\mathcal{A}=(\mathcal{A} \mathcal{A})_{v}$ and with $\operatorname{mor}(\operatorname{mor}(\mathcal{A A}))$ equal to the class of commutative squares in $\mathcal{A}$. More generally, if $\mathcal{A}$ is a subcategory of another category $\mathcal{B}$ containing all objects of $\mathcal{B}$, then we can form a double category $\mathcal{A B}$ 
such that $(\mathcal{A B})_{h}=\mathcal{B},(\mathcal{A B})_{v}=\mathcal{A}$, and such that $\operatorname{mor}(\operatorname{mor}(\mathcal{A B}))$ is the class of commutative squares in $\mathcal{B}$ whose vertical arrows belong to the subcategory $\mathcal{A}$ :

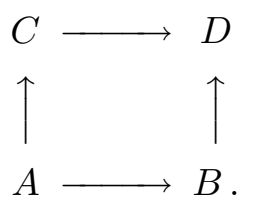

3.3 Lemma $[22$, Lemma 1.6.5] The inclusion of nerves $|\mathcal{B}| \rightarrow|\mathcal{A B}|$ is a homotopy equivalence.

Recall that the homotopy limit of a cofunctor $F$ from a small (ordinary) category $\mathcal{C}$ to $\mathcal{T}$, the category of Spaces, is the totalization of the cosimplicial Space

$$
p \mapsto \prod_{G:[p] \rightarrow \mathcal{C}} F(G(0))
$$

where the product is taken over all functors $G$ from $[p]=\{0,1, \ldots, p\}$ to $\mathcal{C}$. What can we do if $\mathcal{C}$ is a double category and $F$ is a (double) cofunctor from $\mathcal{C}$ to $\mathcal{T} \mathcal{T}$ ? Then we define the homotopy limit as the totalization of the bicosimplicial Space

$$
(p, q) \mapsto \prod_{H:[p] \times[q] \rightarrow \mathcal{C}} F(G(0,0))
$$

Note that $[p] \times[q]$ is a double category, horizontal arrows being those which do not change the second coordinate and vertical arrows being those which do not change the first coordinate.

We need a variation on 3.3 involving homotopy limits. In the situation of 3.3, assume that $F$ is a cofunctor from $\mathcal{B}$ to Spaces $(=\mathcal{T})$ taking all morphisms in $\mathcal{A}$ to homotopy equivalences. We can think of $F$ as a double cofunctor from $\mathcal{A B}$ to $\mathcal{T} \mathcal{T}$.

3.4 Lemma The projection

$$
\operatorname{holim}_{\mathcal{A B}} F \rightarrow \underset{\mathcal{B}}{\operatorname{holim}} F
$$

is a homotopy equivalence. 
Proof Let $\mathcal{A}_{p} \mathcal{B}$ be the ordinary category whose objects are diagrams of the form $A_{0} \rightarrow \cdots \rightarrow A_{p}$ in $\mathcal{A}$, with natural transformations in $\mathcal{B}$ between such diagrams as morphisms. It is enough to show that the face functor

$$
d:\left(A_{0} \rightarrow \cdots \rightarrow A_{p}\right) \mapsto A_{0}
$$

induces a homotopy equivalence

$$
d^{*}: \operatorname{holim}_{\mathcal{B}} F \longrightarrow \operatorname{holim}_{\mathcal{A}_{p} \mathcal{B}} F d .
$$

The face functor $d$ has an obvious left adjoint, say $e$. Thus there is a natural transformation $\tau$ from $e d$ to the identity on $\mathcal{A}_{p} \mathcal{B}$. The natural transformation is a functor

$$
\tau:[1] \times \mathcal{A}_{p} \mathcal{B} \longrightarrow \mathcal{A}_{p} \mathcal{B} .
$$

Now the key observation is that $F d \tau$ equals the composition

$$
[1] \times \mathcal{A}_{p} \mathcal{B} \stackrel{\text { projection }}{\longrightarrow} \mathcal{A}_{p} \mathcal{B} \stackrel{F d}{\longrightarrow} \mathcal{T} \text {. }
$$

Hence $\tau^{*}$ can be defined as a map from holim $F d$ to $\operatorname{holim}(F d \cdot \operatorname{proj})$. Now $i_{0}^{*} \tau^{*}=(e d)^{*}$ and $i_{1}^{*} \tau^{*}=\mathrm{id}$, where $i_{0}$ and $i_{1}$ are the standard injections of $\mathcal{A}_{p} \mathcal{B}$ in $[1] \times \mathcal{A}_{p} \mathcal{B}$. Therefore $(e d)^{*}$ is homotopic to the identity. Also, de is an identity functor.

To be more specific now, let $\mathcal{I} k \subset \mathcal{O} k$ be the subcategory consisting of all morphisms which are isotopy equivalences. Eventually we will be interested in the double category $\mathcal{I} k \mathcal{O} k$. Right now we need a lemma concerning $\mathcal{I} k$ itself.

\subsection{Lemma}

$$
|\mathcal{I} k| \simeq \coprod_{0 \leq j \leq k}\left(\begin{array}{c}
M \\
j
\end{array}\right)
$$

Proof Observe that $\mathcal{I} k$ is a coproduct $\coprod_{\mathcal{I}^{(j)}}$ where $0 \leq j \leq k$ and the objects of $\mathcal{I}^{(j)}$ are the open subsets of $M$ diffeomorphic to a union of $j$ copies of $\mathbb{R}^{m}$. We have to show

$$
\left|\mathcal{I}^{(j)}\right| \simeq\left(\begin{array}{c}
M \\
j
\end{array}\right)
$$

For $j=0$ this is obvious. Here is a proof for $j=1$, following [5, 3.1]. Let $E \subset\left|\mathcal{I}^{(1)}\right| \times M$ consist of all pairs $(x, y)$ such that the (open) cell of $\left|\mathcal{I}^{(1)}\right|$ containing $x$ corresponds to a nondegenerate simplex (diagram in $\mathcal{I}^{(1)}$ )

$$
V_{0} \rightarrow V_{1} \rightarrow \cdots \rightarrow V_{r}
$$


where $y \in V_{r}$. The projection maps

$$
\left|\mathcal{I}^{(1)}\right| \leftarrow E \rightarrow M
$$

are almost locally trivial in the sense of [20, A.1], since $E$ is open in $\left|\mathcal{I}^{(1)}\right| \times M$. By $[20$, A.2] it is enough to verify that both have contractible fibers. Each fiber of $E \rightarrow\left|\mathcal{I}^{(1)}\right|$ is homeomorphic to euclidean space $\mathbb{R}^{n}$.

Let $E_{y}$ be the fiber of $E \rightarrow M$ over $y \in M$. This embeds in $\left|\mathcal{I}^{(1)}\right|$ under the projection, and we can describe it as the union of all open cells corresponding to nondegenerate simplices $\left(U_{0} \rightarrow \cdots \rightarrow U_{k}\right)$ where $U_{k}$ contains $y$. There is a subspace $D_{y} \subset E_{y}$ defined as the union of all open cells corresponding to nondegenerate simplices $\left(U_{0} \rightarrow \cdots \rightarrow U_{k}\right)$ where $U_{0}$ contains $y$. Note the following:

- $D_{y}$ is a deformation retract of $E_{y}$. Namely, suppose that $x$ in $E_{y}$ belongs to a cell corresponding to a simplex $\left(U_{0}, \ldots, U_{k}\right)$ with $y \in U_{k}$. Let $\left(x_{0}, x_{1}, \ldots, x_{k}\right)$ be the barycentric coordinates of $x$ in that simplex, all $x_{i}>0$, and let $j \leq k$ be the least integer such that $y \in U_{j}$. Define a deformation retraction by

$$
\begin{aligned}
h_{1-t}(x):= & \left(t x_{\mathrm{no}}+x_{\mathrm{yes}}\right)^{-1}\left(t x_{0}, \ldots, t x_{j-1}, x_{j}, \ldots, x_{k}\right) \\
& x_{\mathrm{no}}:=\sum_{i<j} x_{i} \quad x_{\mathrm{yes}}:=\sum_{i \geq j} x_{i}
\end{aligned}
$$

for $t \in[0,1]$, using the barycentric coordinates in the same simplex.

- $D_{y}$ is homeomorphic to the classifying space of the poset of all $U \in \mathcal{I}^{(1)}$ containing $y$. The opposite poset is directed, so $D_{y}$ is contractible.

Hence $E_{y}$ is contractible, and the proof for $j=1$ is complete. In the general case $j \geq 1$ let

$$
E \subset \mathcal{I}^{(j)} \times\left(\begin{array}{c}
M \\
j
\end{array}\right)
$$

consist of all pairs $(x, S)$ such that the (open) cell of $\left|\mathcal{I}^{(j)}\right|$ containing $x$ corresponds to a nondegenerate simplex

$$
V_{0} \rightarrow V_{1} \rightarrow \cdots \rightarrow V_{r}
$$

(diagram in $\mathcal{I}^{(j)}$ ) where each component of $V_{r}$ contains exactly one point from $S$. Again the projections from $E$ to $\left|\mathcal{I}^{(j)}\right|$ and to $\left(\begin{array}{c}M \\ j\end{array}\right)$ are homotopy equivalences. 
For $p \geq 0$ let $\mathcal{I} k \mathcal{O} k_{p}$ be the category whose objects are functors $G:[p] \rightarrow \mathcal{O} k$ and whose morphisms are double functors

$$
[1] \times[p] \longrightarrow \mathcal{I} k \mathcal{O} k \text {. }
$$

(Note that the nerve of the simplicial category $p \mapsto \mathcal{I} k \mathcal{O} k_{p}$ is isomorphic to the nerve of the double category $\mathcal{I} k \mathcal{O} k$.) The rule $G \mapsto G(p)$ is a functor from $\mathcal{I} k \mathcal{O} k_{p}$ to $\mathcal{I} k$. In the next lemma we have to make explicit reference to $M$ and another manifold $V$, so we write $\mathcal{O} k(M), \mathcal{I} k(M)$ and so on.

3.6 Lemma For any object $V$ in $\mathcal{I} k(M)$, the homotopy fiber over the 0 simplex $V$ of the map

$$
\left|\mathcal{I} k \mathcal{O} k_{p}(M)\right| \longrightarrow|\mathcal{I} k(M)|
$$

induced by $G \mapsto G(p)$ is homotopy equivalent to $\left|\mathcal{I} k \mathcal{O} k_{p-1}(V)\right|$.

3.7 Remark Combining 3.6 and 3.5, and induction on $p$, we can get a very good idea of the homotopy type of $\left|\mathcal{I} k \mathcal{O} k_{p}(M)\right|$. In particular, the functor

$$
V \mapsto\left|\mathcal{I} k \mathcal{O} k_{p}(V)\right|
$$

from $\mathcal{O}=\mathcal{O}(M)$ to Spaces takes isotopy equivalences to homotopy equivalences because the functors $V \mapsto\left(\begin{array}{c}V \\ j\end{array}\right)$ have this property.

Proof of 3.6 Using Thomason's homotopy colimit theorem [21] we can make the identification

$$
\left|\mathcal{I} k \mathcal{O} k_{p}(M)\right| \simeq \operatorname{hocolim}_{V \in \mathcal{I} k(M)}\left|\mathcal{I} k \mathcal{O} k_{p-1}(V)\right| .
$$

Then the map under investigation corresponds to the projection from the homotopy colimit to the nerve of $\mathcal{I} k(M)$. This map is already a quasifibration of simplicial sets. Namely, all morphisms $V_{1} \rightarrow V_{2}$ in $\mathcal{I} k(M)$ are isotopy equivalences by definition, and inductively we may assume that the functor $V \mapsto\left|\mathcal{I} k \mathcal{O} k_{p-1}(V)\right|$ takes isotopy equivalences to homotopy equivalences (see remark 3.7). Therefore the homotopy fiber that we are interested in has the same homotopy type as the honest fiber.

Let $E$ be a cofunctor from $\mathcal{O} k=\mathcal{O}_{k}(M)$ to Spaces taking morphisms in $\mathcal{O} k$ which are isotopy equivalences to homotopy equivalences. Use this to define a

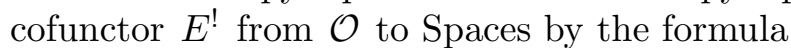

$$
E^{!}(V)=\operatorname{holim}_{U \in \mathcal{O} k(V)} E(U) .
$$

In categorical patois: $E^{!}$is the homotopy right Kan extension of $E$ along the inclusion functor $\mathcal{O} k \rightarrow \mathcal{O}$. 
3.8 Lemma $E^{!}$is good.

Proof From 3.4 we know that the projection

$$
\operatorname{holim}_{U \in \mathcal{I} k \mathcal{O} k(V)} E(U) \longrightarrow \operatorname{holim}_{U \in \mathcal{O} k(V)} E(U)
$$

is a homotopy equivalence. The domain of this projection can be thought of as the totalization of the cosimplicial Space

$$
p \mapsto \underset{U_{0} \rightarrow \cdots \rightarrow U_{p}}{\operatorname{holim}} E\left(U_{0}\right)
$$

where the homotopy limit, holim $E\left(U_{0}\right)$, is taken over $\mathcal{I} k \mathcal{O} k_{p}(V)$ as defined just before 3.6. Note that the cofunctor $\left(U_{0} \rightarrow \cdots \rightarrow U_{p}\right) \mapsto E\left(U_{0}\right)$ takes all morphisms to homotopy equivalences. Hence its homotopy colimit is quasifibered over the nerve of the indexing category, and its homotopy limit may be identified (up to homotopy equivalence) with the section Space of the associated fibration. Using 3.6 and 3.7 now we see that

$$
V \mapsto \operatorname{holim}_{U_{0} \rightarrow \cdots \rightarrow U_{p}} E\left(U_{0}\right)
$$

is a good cofunctor $E_{p}^{!}$for each $p$. Hence $E^{!}$is good, too.

We come to the main result of the section. It is similar to certain well-known statements about small simplices, for example [2, III.7.3], which are commonly used to prove excision theorems. Let $\varepsilon$ be an open cover of $M$. We say that $V \in \mathcal{O} k$ is $\varepsilon$-small if each connected component of $V$ is contained in some open set of the cover $\varepsilon$. Let $\varepsilon \mathcal{O} k=\varepsilon \mathcal{O} k(M)$ be the full sub-poset of $\mathcal{O} k$ consisting of the $\varepsilon$-small objects. For $V \in \mathcal{O}$ let

$$
\varepsilon E^{!}(V):=\operatorname{holim}_{U \in \varepsilon \mathcal{O} k(V)} E(U) .
$$

3.9 Theorem The projection $E^{!}(V) \rightarrow \varepsilon E^{!}(V)$ is a homotopy equivalence.

Proof Using the notation from the proof of 3.8, and obvious $\varepsilon$-modifications, we see that it suffices to prove that the projection $E_{p}^{!}(V) \rightarrow \varepsilon E_{p}^{!}(V)$ is a homotopy equivalence, for all $V$ and $p$. However, the analysis of $E_{p}^{!}(V)$ as a section Space (proof of 3.8) works equally well for $\varepsilon E_{p}^{!}(V)$, and gives the same result up to homotopy equivalence. In particular 3.5 and 3.6 go through in the $\varepsilon$-setting. 


\section{Construction of Polynomial Cofunctors}

We continue to assume that $E$ is a cofunctor from $\mathcal{O} k$ to Spaces taking isotopy equivalences to homotopy equivalences.

4.1 Theorem The cofunctor $E^{!}$on $\mathcal{O}$ is polynomial of degree $\leq k$.

Proof We have to verify that the condition in 2.2 is satisfied. Without loss of generality, $V=M$. Then $A_{0}, A_{1}, \ldots, A_{k}$ are pairwise disjoint closed subsets of $M$. Let $M_{i}=M \backslash A_{i}$ and $M_{S}=\cap_{i \in S} M_{i}$ for $S \subset\{0,1, \ldots, k\}$. Using 3.9, all we have to show is that the $(k+1)$-cube of Spaces

$$
S \mapsto \varepsilon E^{!}\left(M_{S}\right)
$$

is homotopy Cartesian. Here $\varepsilon$ can be any open cover of $M$, and in the present circumstances we choose it so that none of the open sets in $\varepsilon$ meets more than one $A_{i}$. Then

$$
\varepsilon \mathcal{O} k=\bigcup_{i} \varepsilon \mathcal{O} k\left(M_{i}\right)
$$

(This is the pigeonhole principle again: Each component of an object $U$ in $\varepsilon \mathcal{O}_{k}$ meets at most one of the $A_{i}$, but since $U$ has at most $k$ components, $U \cap A_{i}=\emptyset$ for some $i$.) With lemma 4.2 below, we conclude that the canonical map

$$
\underset{\varepsilon \mathcal{O} k}{\operatorname{holim}} E \longrightarrow \operatorname{holim}_{S \neq \emptyset} \operatorname{holim}_{\varepsilon \mathcal{O} k\left(M_{S}\right)} E
$$

is a homotopy equivalence. But this is what we had to show.

In lemma 4.2 just below, an ideal in a poset $\mathcal{Q}$ is a subset $\mathcal{R}$ of $\mathcal{Q}$ such that for every $b \in \mathcal{R}$, all $a \in \mathcal{Q}$ with $a \leq b$ belong to $\mathcal{R}$.

4.2 Lemma Suppose that the poset $\mathcal{Q}$ is a union of ideals $\mathcal{Q}_{i}$, where $i \in T$. For finite nonempty $S \subset T$ let $\mathcal{Q}_{S}=\cap_{i \in S} \mathcal{Q}_{i}$. Let $E$ be a cofunctor from $\mathcal{Q}$ to Spaces. Then the canonical map

$$
\underset{\mathcal{Q}}{\operatorname{holim}} E \longrightarrow \operatorname{holim}_{S \neq \emptyset} \operatorname{holim}_{\mathcal{Q}_{S}} E
$$

is a homotopy equivalence. 
Proof Let $\int \mathcal{Q}_{S}$ be the poset consisting of all pairs $(S, x)$ where $S \subset T$ is finite, nonempty and where $(S, x) \leq\left(S^{\prime}, y\right)$ if and only if $S \subset S^{\prime}$ and $x \leq y$ in $\mathcal{Q}$. The forgetful map taking $(S, x)$ to $x$ is a functor $u: \int \mathcal{Q}_{S} \rightarrow \mathcal{Q}$. It is right cofinal, ie the under category $y \downarrow u$ is contractible for every $y$ in $\mathcal{Q}$. Therefore, by the cofinality theorem for homotopy inverse limits [1, ch. XI, 9.2], [4, 9.3] the obvious map

$$
\operatorname{holim}_{x \in \mathcal{Q}} E(x) \longrightarrow \underset{(S, x) \in \int \mathcal{Q}_{S}}{\operatorname{holim}} E(x)
$$

is a homotopy equivalence. (Note that it has to be right cofinal instead of the usual left cofinal because we are dealing with a cofunctor $E$.) By inspection, the codomain of this map is homeomorphic to

$$
\underset{S \neq \emptyset}{\operatorname{holim}} \operatorname{holim}_{\mathcal{Q}_{S}} E \text {. }
$$

Remark Note that the obvious map $E(U) \rightarrow E^{!}(U)$ is a homotopy equivalence for every $U$ in $\mathcal{O} k$. This is again an application of the cofinality theorem for homotopy inverse limits, although a much more obvious one. In this sense $E^{!}$extends $E$.

\section{Characterizations of Polynomial Cofunctors}

5.1 Theorem Let $\gamma: F_{1} \rightarrow F_{2}$ be a morphism in $\mathcal{F}$. Suppose that both $F_{1}$ and $F_{2}$ are polynomial of degree $\leq k$. If $\gamma: F_{1}(V) \rightarrow F_{2}(V)$ is a homotopy equivalence for all $V \in \mathcal{O} k$, then it is a homotopy equivalence for all $V \in \mathcal{O}$.

Proof Suppose that $\gamma: F_{1}(V) \rightarrow F_{2}(V)$ is a homotopy equivalence for all $V \in \mathcal{O} k$. Suppose also that $W \in \mathcal{O} r$, where $r>k$. Let $A_{0}, A_{1}, \ldots, A_{k}$ be distinct components of $W$ and let $W_{S}=\cap_{i \in S}\left(W \backslash A_{i}\right)$ for $S \subset\{0,1, \ldots, k\}$. Then

$$
F_{i}(W) \simeq \operatorname{holim}_{S \neq 0} F_{i}\left(W_{S}\right)
$$

for $i=1,2$ and therefore $\gamma: F_{1}(W) \rightarrow F_{2}(W)$ is a homotopy equivalence provided $\gamma$ from $F_{1}\left(W_{S}\right) \rightarrow F_{2}\left(W_{S}\right)$ is a homotopy equivalence for all nonempty $S \subset\{0,1, \ldots, k\}$. But $W_{S}$ for $S \neq \emptyset$ has fewer components than $W$, so by induction the proviso is correct. This takes care of all $W \in \cup_{r} \mathcal{O} r$.

Next, suppose that $W=\operatorname{int}(L)$ where $L$ is a smooth compact codimension zero submanifold of $M$. Choose a handle decomposition for $L$, let $s$ be the maximum of the indices of the handles, and let $t$ be the number of handles of 
index $s$ that occur. If $s=0$ we have $W \in \mathcal{O} r$ for some $r$ and this case has been dealt with. If $s>0$, let $e: \mathbb{D}^{m-s} \times \mathbb{D}^{s} \rightarrow L$ be one of the $s$-handles. We assume that $e^{-1}(\partial L)$ is $\partial \mathbb{D}^{m-s} \times \mathbb{D}^{s}$. Since $s>0$ we can find pairwise disjoint small closed disks $C_{0}, \ldots, C_{k}$ in $\mathbb{D}^{s}$ and we let

$$
A_{i}:=e\left(\mathbb{D}^{m-s} \times C_{i}\right) \cap W
$$

for $0 \leq i \leq k$. Then each $A_{i}$ is closed in $W$ and $W \backslash A_{i}$ is the interior of a smooth handlebody in $M$ which has a handle decomposition with no handles of index $>s$, and fewer than $t$ handles of index $s$. The same is true for $W_{S}:=\cap_{i \in S}\left(W \backslash A_{i}\right)$ provided $S \neq \emptyset$. Therefore, by induction,

$$
\gamma: F_{1}\left(W_{S}\right) \longrightarrow F_{2}\left(W_{S}\right)
$$

is a homotopy equivalence for $\emptyset \neq S \subset\{0,1, \ldots, k\}$ and consequently the right-hand vertical arrow in

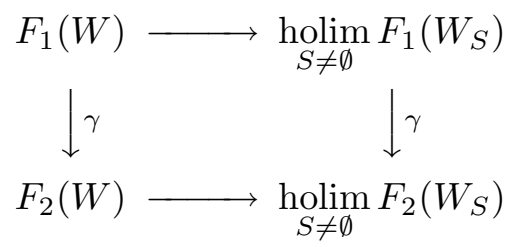

is a homotopy equivalence. But the two horizontal arrows are also homotopy equivalences, because $F_{1}$ and $F_{2}$ are polynomial of degree $\leq k$. Therefore the left-hand vertical arrow is a homotopy equivalence. This takes care of every $W \in \mathcal{O}$ which is the interior of a compact smooth handlebody in $M$.

The general case follows because $F_{1}$ and $F_{2}$ are good cofunctors; see especially property (b) in the definition of goodness, just after 1.1.

For $F$ in $\mathcal{F}$ let $T_{k} F$ be the homotopy right Kan extension of the restriction of $F$ to $\mathcal{O} k$. The explicit formula is

$$
T_{k} F(V):=\operatorname{holim}_{U \in \mathcal{O} k(V)} F(U) .
$$

From section 3 and section 4 we know that $T_{k} F$ is good and polynomial of degree $\leq k$. There is an obvious forgetful morphism $\eta_{k}: F \rightarrow T_{k} F$. Clearly the natural map $\eta_{k}: F(U) \rightarrow T_{k} F(U)$ is a homotopy equivalence for every $U \in \mathcal{O} k$. Hence, by 5.1, if $F$ is already polynomial of degree $\leq k$, then $\eta_{k}$ from $F(V)$ to $T_{k} F(V)$ is a homotopy equivalence for every $V \in \mathcal{O}$. In this sense an $F$ which is polynomial of degree $\leq k$ is determined by its restriction $E$ to $\mathcal{O} k$. The restriction does of course take isotopy equivalences in $\mathcal{O} k$ to homotopy equivalences. We saw in section 4 that that is essentially the only condition it must satisfy.

The polynomial objects in $\mathcal{F}$ can also be characterized in sheaf theoretic terms. Recall the Grothendieck topologies $\mathcal{J}_{k}$ on $\mathcal{O}$, from the introduction. 
5.2 Theorem A good cofunctor $F$ from $\mathcal{O}$ to Spaces is polynomial of degree $\leq k$ if and only if it is a homotopy sheaf with respect to the Grothendieck topology $\mathcal{J}_{k}$.

Proof Suppose that $F$ is a homotopy sheaf with respect to $\mathcal{J}_{k}$. Let $V \in \mathcal{O}$ and pairwise disjoint closed subsets $A_{0}, \ldots, A_{k}$ of $V$ be given. Let $V_{i}=V \backslash A_{i}$. Then the inclusions $V_{i}$ for $0 \leq i \leq k$ form a covering of $V$ in the Grothendieck topology $\mathcal{J}_{k}$. Hence

$$
F(V) \longrightarrow \operatorname{holim}_{R} F\left(\cap_{i \in R} V_{i}\right)
$$

is a homotopy equivalence; the homotopy limit is taken over the nonempty subsets $R$ of $\{0, \ldots, k\}$. This shows that $F$ is polynomial of degree $\leq k$.

Conversely, suppose that $F$ is polynomial of degree $\leq k$. Let $W \in \mathcal{O}$ be given and let $\left\{V_{i} \rightarrow W \mid i \in S\right\}$ be a covering of $W$ in the Grothendieck topology $\mathcal{J}_{k}$. Let $E$ be the restriction of $F$ to $\mathcal{O} k$. Define $\varepsilon E^{!}$as in section 3, just before 3.9 , where $\varepsilon$ is the covering $\left\{V_{i}\right\}$. Up to equivalence, $F$ and $\varepsilon E^{!}$are the same. By 4.2, the canonical map

$$
\varepsilon E^{!}(W) \longrightarrow \operatorname{holim}_{R} \varepsilon E^{!}\left(\cap_{i \in R} V_{i}\right)
$$

is a homotopy equivalence. Here again, $R$ runs through the finite nonempty subsets $R$ of $S$.

\section{Approximation by Polynomial Cofunctors}

From section 5 , we have for every $k \geq 0$ an endofunctor $T_{k}: \mathcal{F} \rightarrow \mathcal{F}$ given by the rule $F \mapsto T_{k} F$, and a natural transformation from the identity $\mathcal{F} \rightarrow \mathcal{F}$ to $T_{k}$ given by $\eta_{k}: F \rightarrow T_{k} F$ for all $F$. It is sometimes convenient to define $T_{-1}$ as well, by $T_{-1} F(V):=*$. The following theorem is mostly a summary of results from section 5 . It tries to say that $T_{k}$ is essentially left adjoint to the inclusion functor $\mathcal{F}_{k} \rightarrow \mathcal{F}$. Here $\mathcal{F}_{k}$ is the full subcategory of $\mathcal{F}$ consisting of the objects which are polynomial of degree $\leq k$. Compare [25, Thm.6.1].

6.1 Theorem The following holds for every $F$ in $\mathcal{F}$ and every $k \geq 0$.

(1) $T_{k} F$ is polynomial of degree $\leq k$.

(2) If $F$ is already polynomial of degree $\leq k$, then $\eta_{k}: F \rightarrow T_{k} F$ is an equivalence.

(3) $T_{k}\left(\eta_{k}\right): T_{k} F \rightarrow T_{k}\left(T_{k} F\right)$ is an equivalence. 
Proof Properties (1) and (2) have been established in section 5. As for (3), we can use 5.1 and we then only have to verify that

$$
T_{k}\left(\eta_{k}\right): T_{k} F(W) \rightarrow T_{k}\left(T_{k} F(W)\right)
$$

is a homotopy equivalence for every $W \in \mathcal{O} k$. Written out in detail the map takes the form

$$
\begin{aligned}
\operatorname{holim}_{V \in \mathcal{O} k(W)} F(V) & \longrightarrow \operatorname{holim}_{V \in \mathcal{O} k(W)} T_{k} F(V) \\
& =\operatorname{holim}_{V \in \mathcal{O} k(W)} \operatorname{holim}_{U \in \mathcal{O} k(V)} F(U)
\end{aligned}
$$

and it is induced by the maps $F(V) \rightarrow \operatorname{holim}_{U} F(U)$ for $V$ in $\mathcal{O} k(V)$. These maps are clearly homotopy equivalences, since the identity morphism $V \rightarrow V$ is a terminal object in $\mathcal{O} k(V)$.

Remark One way of saying that the inclusion of a full subcategory, say $\mathcal{A} \rightarrow$ $\mathcal{B}$, has a left adjoint is to say that there exists a functor $T: \mathcal{B} \rightarrow \mathcal{B}$ and a natural transformation $\eta: \operatorname{id}_{\mathcal{B}} \rightarrow T$ with the following properties.

(1) $T(b)$ belongs to $\mathcal{A}$ for every $b$ in $\mathcal{B}$.

(2) For $a$ in $\mathcal{A}$, the morphism $\eta: a \rightarrow T(a)$ is an isomorphism.

(3) For $b$ in $\mathcal{B}$, the morphism $T(\eta): T(b) \rightarrow T(T(b))$ is an isomorphism.

From the definitions, there are forgetful transformations $r_{k}: T_{k} F \rightarrow T_{k-1} F$ for any $F$ and any $k>0$. They satisfy the relations $r_{k} \eta_{k}=\eta_{k-1}: F \rightarrow T_{k-1} F$. Therefore

$$
\left\{\eta_{k}\right\}: F \longrightarrow \operatorname{holim}_{k} T_{k} F
$$

is defined. The codomain, with its inverse filtration, may be called the Taylor tower of $F$. Usually one wants to know whether $(*)$ is a homotopy equivalence. More precisely one can ask two questions:

- Does the Taylor tower of $F$ converge?

- If it does converge, does it converge to $F$ ?

Regarding the first question: although $\operatorname{holim}_{k} T_{k} F$ is always defined, we would not speak of convergence unless the connectivity of $r_{k}: T_{k} F(V) \rightarrow T_{k-1} F(V)$ tends to infinity with $k$, independently of $V$. 


\section{More Examples of Polynomial Cofunctors}

7.1 Example Let $p: Z \rightarrow\left(\begin{array}{c}M \\ k\end{array}\right)$ be a fibration. For $U \subset\left(\begin{array}{c}M \\ k\end{array}\right)$ let $\Gamma(p ; U)$ be the Space of partial sections of $p$ defined over $U$. The cofunctor $F$ on $\mathcal{O}$ defined by $F(V):=\Gamma\left(p ;\left(\begin{array}{l}V \\ k\end{array}\right)\right)$ is good and, moreover, it is polynomial of degree $\leq k$. This can be proved like 2.4 .

Keep the notation of 7.1. Let $\boldsymbol{\Delta}_{k} V$ be the complement of $\left(\begin{array}{l}V \\ k\end{array}\right)$ in the $k$-fold symmetric power $\operatorname{sp}_{k} V:=(V \times V \times \ldots \times V) / \Sigma^{k}$. The homotopy colimit in the next lemma is taken over the poset of all neighborhoods $Q$ of $\boldsymbol{\Delta}_{k} V$ in $\operatorname{sp}_{k} V$.

7.2 Lemma The cofunctor $G$ on $\mathcal{O}$ given by

$$
G(V):=\underset{Q}{\operatorname{hocolim}} \Gamma\left(p ;\left(\begin{array}{l}
V \\
k
\end{array}\right) \cap Q\right)
$$

is good.

Proof We concentrate on part (b) of goodness to begin with. Fix $V$ and choose a smooth triangulation on the $k$-fold product $(V)^{k}$, equivariant with respect to the symmetric group $\Sigma_{k}$. Then $\operatorname{sp}_{k} V$ has a preferred PL structure and $\boldsymbol{\Delta}_{k} V$ is a PL subspace, so we can speak of regular neighborhoods of $\boldsymbol{\Delta}_{k} V$. It is clear that all regular neighborhoods of $\boldsymbol{\Delta}_{k} V$ have the same homotopy type, and that each neighborhood of $\boldsymbol{\Delta}_{k} V$ contains a regular one. Therefore, if $L$ is a regular neighborhood of $\boldsymbol{\Delta}_{k} V$, then the canonical inclusion

$$
\Gamma\left(p ;\left(\begin{array}{l}
V \\
k
\end{array}\right) \cap \operatorname{int}(L)\right) \longrightarrow \underset{Q}{\operatorname{hocolim}} \Gamma\left(p ;\left(\begin{array}{l}
V \\
k
\end{array}\right) \cap Q\right)
$$

is a homotopy equivalence. This observation tends to simplify matters. Another observation which tends to complicate matters is that for an open subset $U$ of $V$ and a regular neighborhood $L$ as above, the intersection of $L$ with $\operatorname{sp}_{k} U$ will usually not be a regular neighborhood of $\boldsymbol{\Delta}_{k} U$. However, we can establish goodness as follows. Suppose that

$$
V=\cup_{i} K_{i}
$$

where each $K_{i}$ is a smooth compact codimension zero submanifold of $V$, and $K_{i} \subset \operatorname{int}\left(K_{i+1}\right)$. As in the proof of 1.4 , it is enough to show that the canonical map

$$
G(V) \longrightarrow \operatorname{holim}_{i} G\left(\operatorname{int}\left(K_{i}\right)\right)
$$

is a homotopy equivalence. Abbreviate $\operatorname{int}\left(K_{i}\right)=V_{i}$. Choose a regular neighborhood $L$ of $\boldsymbol{\Lambda}_{k} V$ in $\operatorname{sp}_{k} V$ such that $L \cap \mathrm{sp}_{k}\left(K_{i}\right)$ is a regular neighborhood of $\boldsymbol{\Delta}_{k}\left(K_{i}\right)$ in $\operatorname{sp}_{k}\left(K_{i}\right)$ for each $i$. Then it is not hard to see that the inclusion

$$
\Gamma\left(p ;\left(\begin{array}{c}
V_{i} \\
k
\end{array}\right) \cap \operatorname{int}(L)\right) \longrightarrow \underset{R}{\operatorname{hocolim}} \Gamma\left(p ;\left(\begin{array}{c}
V_{i} \\
k
\end{array}\right) \cap R\right)
$$


is a homotopy equivalence, for each $i$. Therefore, in the commutative diagram

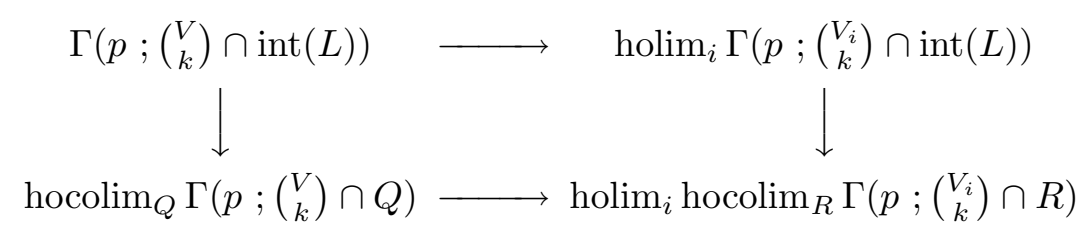

the two vertical arrows are homotopy equivalences. The upper horizontal arrow is also a homotopy equivalence by inspection. Hence the lower horizontal arrow is a homotopy equivalence. This completes the proof of part (b) of goodness.

Proof of part (a) of goodness: Suppose that $W \hookrightarrow V$ in $\mathcal{O}$ is an isotopy equivalence. Let $\left\{j_{t}: V \rightarrow V\right\}$ be a smooth isotopy of embeddings, with $j_{0}=$ $\operatorname{id}_{V}$ and $\operatorname{im}\left(j_{1}\right)=W$. Let

$$
X:=\underset{R}{\operatorname{hocolim}} \Gamma\left(j^{*} p ;\left(\left(\begin{array}{l}
V \\
k
\end{array}\right) \times I\right) \cap R\right)
$$

where $I=[0,1]$ and $j^{*} p$ is the pullback of $p$ under the map

$$
\left(\begin{array}{l}
V \\
k
\end{array}\right) \times I \longrightarrow\left(\begin{array}{l}
V \\
k
\end{array}\right) \quad ; \quad(S, t) \mapsto j_{t}(S)
$$

and $R$ runs over the neighborhoods of $\boldsymbol{\Lambda}_{k} V \times I$ in $\operatorname{sp}_{k} V \times I$. Key observation: Every $R$ contains a neighborhood of the form $Q \times I$, where $Q \subset \operatorname{sp}_{k} V$. This implies that the restriction maps

$$
G(W) \stackrel{\rho_{W}}{\longleftarrow} X \stackrel{\rho_{V}}{\longrightarrow} G(V)
$$

(induced by the bundle maps $j_{1}^{*} p \rightarrow j^{*} p \leftarrow j_{0}^{*} p$ ) are homotopy equivalences. The restriction map $G(V) \rightarrow G(W)$ that we are interested in can be written as a composition

$$
G(V) \stackrel{j^{*}}{\longrightarrow} X \stackrel{\rho_{W}}{\longrightarrow} G(W)
$$

where the arrow labelled $j^{*}$ is right inverse to $\rho_{V}$. Therefore the restriction map $G(V) \rightarrow G(W)$ is a homotopy equivalence.

7.3 Lemma The cofunctor $G$ in 7.2. is polynomial of degree $\leq k$.

Proof Fix $W \in \mathcal{O}$ and let $A_{0}, \ldots, A_{k}$ be closed and pairwise disjoint in $W$. Let $W_{i}:=W \backslash A_{i}$ and choose neighborhoods $Q_{i}$ of $\boldsymbol{\Delta}_{k} W_{i}$ in $\operatorname{sp}_{k} W_{i}$. Let

$$
\begin{aligned}
W_{S} & =\cap_{i \in S} W_{i} \\
Q_{S} & =\cap_{i \in S} Q_{i}
\end{aligned}
$$


for nonempty $S \subset\{0,1, \ldots, k\}$, and $W_{\emptyset}=W, Q_{\emptyset}=\cup_{i} Q_{i}$. Then

$$
\left(\begin{array}{c}
W \\
k
\end{array}\right) \cap Q_{\emptyset}=\bigcup_{i}\left(\begin{array}{c}
W_{i} \\
k
\end{array}\right) \cap Q_{i} \simeq \underset{S \neq \emptyset}{\operatorname{hocolim}}\left(\begin{array}{c}
W_{S} \\
k
\end{array}\right) \cap Q_{S}
$$

which shows, much as in the proof of 2.4 , that the obvious map

$$
\Gamma\left(p ;\left(\begin{array}{c}
W \\
k
\end{array}\right) \cap Q_{\emptyset}\right) \longrightarrow \operatorname{holim}_{S \neq \emptyset} \Gamma\left(p ;\left(\begin{array}{c}
W_{S} \\
k
\end{array}\right) \cap Q_{S}\right)
$$

is a homotopy equivalence. We can now complete the proof with two observations. Firstly, the neighborhoods of $\boldsymbol{\Lambda}_{k} W_{S}$ of the form $Q_{S}$, as above, form an initial subset [17] in the poset of all neighborhoods. Secondly, there are situations in which homotopy inverse limits commute (up to homotopy equivalence) with homotopy direct limits, and this is one of them. Here we are interested in a double homotopy limit/colimit of the form

$$
\underset{S \neq \emptyset}{\operatorname{holim}} \operatorname{hocolim}_{Q_{0}, \ldots, Q_{k}}(-)
$$

where the blank indicates an expression depending on $S$ and the $Q_{i}$ (actually only on the $Q_{i}$ for $i \in S$ ). Clearly sublemma 7.4 below applies.

7.4 Sublemma Let $X$ be a functor from a product $\mathcal{A} \times \mathcal{B}$ to Spaces, where $\mathcal{A}$ and $\mathcal{B}$ are posets. Suppose that $\mathcal{A}$ is finite and that $\mathcal{B}$ is directed. Then

$$
\underset{b \in \mathcal{B}}{\operatorname{hocolim}} \operatorname{holim}_{a \in \mathcal{A}} X(a, b) \simeq \operatorname{holim}_{a \in \mathcal{A}} \underset{b \in \mathcal{B}}{\operatorname{hocolim}} X(a, b) .
$$

Proof Since $\mathcal{B}$ is a directed poset, the homotopy colimits may be replaced by honest colimits [1]. The universal property of colimits yields a map

$$
\underset{b \in \mathcal{B}}{\operatorname{colim}} \operatorname{holim}_{a \in \mathcal{A}} X(a, b) \simeq \operatorname{holim}_{a \in \mathcal{A}} \operatorname{colim}_{b \in \mathcal{B}} X(a, b)
$$

which is an isomorphism, by inspection.

7.5 Proposition The cofunctor $G$ in 7.2 and 7.3 is in fact polynomial of degree $\leq k-1$.

Proof We must show that $\eta_{k}: G \rightarrow T_{k-1} G$ is an equivalence. Since $G$ and $T_{k-1} G$ are both polynomial of degree $\leq k$, it is enough to check that

$$
\eta_{k}: G(V) \longrightarrow T_{k-1} G(V)
$$

is an equivalence for every $V \in \mathcal{O} k$. See 5.1. If $V$ belongs to $\mathcal{O} r$ for some $r<k$, this is obvious. So we may assume that $V$ has exactly $k$ connected components, 
each diffeomorphic to $\mathbb{R}^{m}$. Denote these components by $A_{0}, \ldots, A_{k-1}$. If we can show that the upper horizontal arrow in

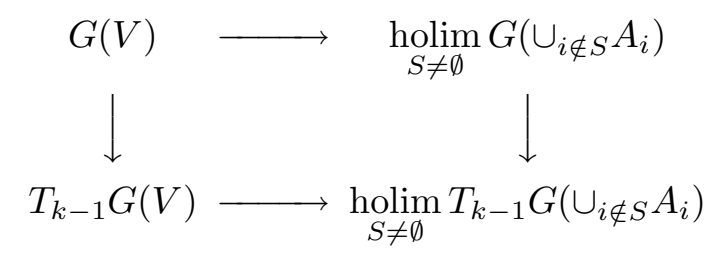

is a homotopy equivalence, then we are done because the lower horizontal and the right-hand vertical arrows are homotopy equivalences. However, this follows in the usual manner (compare proof of 2.4 and of 7.3 ) from the observation that

$$
\left(\begin{array}{l}
V \\
k
\end{array}\right) \cap Q=\bigcup_{i}\left(\begin{array}{c}
V \backslash A_{i} \\
k
\end{array}\right) \cap Q
$$

for sufficiently small neighborhoods $Q$ of $\boldsymbol{\Lambda}_{k} V$ in $\operatorname{sp}_{k} V$. Notice that the observation as such is new because this time the closed subsets $A_{i}$ are $k$ in number, not $k+1$.

We are now in a position to understand the relationship between $F$ in 7.1 and $G$ in 7.2. There is an obvious inclusion $e: F(V) \rightarrow G(V)$, natural in $V$.

7.6 Proposition The morphism $T_{k-1} e: T_{k-1} F \rightarrow T_{k-1} G$ is an equivalence.

Proof By 5.1, it suffices to show that $e: F(V) \rightarrow G(V)$ is a homotopy equivalence for any $V$ which is diffeomorphic to a disjoint union of $\ell$ copies of $\mathbb{R}^{m}$, where $\ell<k$. For such a $V$ choose open subsets

$$
V=V_{0} \supset V_{1} \supset V_{2} \supset V_{3} \supset \ldots
$$

such that the inclusions $V_{i+1} \rightarrow V_{i}$ are isotopy equivalences, such that the closure of $V_{i+1}$ in $V_{i}$ is compact, and such that $\cap_{i} V_{i}$ is a discrete set consisting (necessarily) of $\ell$ points, one in each component of $V$. In the commutative square

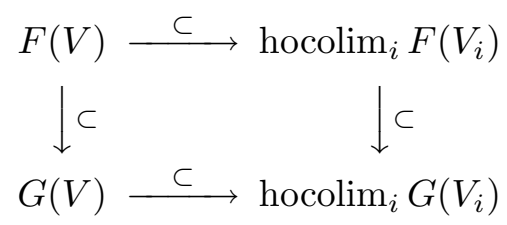

the horizontal arrows are now homotopy equivalences because $F$ and $G$ take isotopy equivalences to homotopy equivalences. On the other hand, suppose that $Q$ is a neighborhood of $\boldsymbol{\Delta}_{k} V_{i}$ in $\operatorname{sp}_{k} V_{i}$ for some $i$. Then clearly there 
exists an integer $j>i$ such that all of $\operatorname{sp}_{k} V_{j}$ is contained in $Q$. It follows that the inclusion of $\operatorname{hocolim}_{i} F\left(V_{i}\right)$ in

$$
\underset{i}{\operatorname{hocolim}} G\left(V_{i}\right)=\underset{i}{\operatorname{hocolim}} \underset{Q}{\operatorname{hocolim}} \Gamma\left(p ;\left(\begin{array}{c}
V_{i} \\
k
\end{array}\right) \cap Q\right)
$$

is a homotopy equivalence. Hence all arrows in $(*)$ are homotopy equivalences.

\section{Homogeneous Cofunctors}

8.1 Definition A cofunctor $E$ in $\mathcal{F}$ is homogeneous of degree $k$, where $k \geq 0$, if it is polynomial of degree $\leq k$ and if $T_{k-1} E(V)$ is contractible for each $V \in \mathcal{O}$.

Remark The cofunctor given by $E(V)=*$ for all $V$ is homogeneous of degree $k$ for any $k \geq 0$. Conversely, if $E$ is homogeneous of degree $k$ and homogeneous of degree $\ell$, where $\ell<k$, then clearly $E(V) \simeq T_{k-1} E(V) \simeq *$.

8.2 Example Let $F$ in $\mathcal{F}$ be arbitrary, and select a point in $F(M)$, if one exists. Then $T_{k} F(V)$ is pointed for all $V$ and $k$. Therefore a new cofunctor $L_{k} F$ can be defined by

$$
L_{k} F(V):=\text { hofiber }\left[T_{k} F(V) \longrightarrow T_{k-1} F(V)\right] .
$$

It follows from 6.1 that $E$ is homogeneous of degree $k$.

8.3 Example Starting with a fibration $p: Z \rightarrow\left(\begin{array}{c}M \\ k\end{array}\right)$, define $F$ as in 7.1 and define $G$ as in 7.2. Select a point in $G(M)$. Then

$$
E(V):=\text { hofiber }[F(V) \stackrel{\subset}{\longrightarrow} G(V)]
$$

is defined. It follows from 7.6 that $E$ is homogeneous of degree $k$.

Example 8.3 deserves to be studied more. Ultimately $E$ has been constructed in terms of the fibration $p$, and a partial section of $p$ defined near the fat diagonal $\boldsymbol{\Delta}_{k} M$. Is it possible to recover $p$ from $E$ ? In particular, for $S \in\left(\begin{array}{c}M \\ k\end{array}\right)$, can we describe the fiber $p^{-1}(S)$ in terms of $E$ ?

Note that $S$ is a subset of $M$ with $k$ elements. Let $V$ be a tubular neighborhood of $S \subset M$, so that $V$ is diffeomorphic to a disjoint union of $k$ copies of $\mathbb{R}^{m}$. Then $S$ belongs to $\left(\begin{array}{c}V \\ k\end{array}\right) \subset\left(\begin{array}{c}M \\ k\end{array}\right)$ and therefore we have maps

$$
E(V) \longrightarrow F(V)=\Gamma\left(p ;\left(\begin{array}{l}
V \\
k
\end{array}\right)\right) \stackrel{\text { evaluation }}{\longrightarrow} p^{-1}(S) .
$$


8.4 Proposition The composite map $E(V) \rightarrow p^{-1}(S)$ is a homotopy equivalence.

Hence we can indeed describe $p^{-1}(S)$ in terms of $E$, up to homotopy equivalence: namely, as $E(V)$ for a tubular neighborhood $V$ os $S$ in $M$.

Proof of 8.4 Much as in the proof of 7.6 we choose a sequence of open subsets

$$
V=V_{0} \supset V_{1} \supset V_{2} \supset V_{3} \supset \ldots
$$

such that the inclusions $V_{i+1} \rightarrow V_{i}$ are isotopy equivalences, such that the closure of $V_{i+1}$ in $V_{i}$ is compact, and such that $\cap_{i} V_{i}=S$. We note that

$$
F(V)=\prod_{j} \Gamma\left(p ; U_{j}\right)
$$

where the $U_{j}$ are the connected components of $\left(\begin{array}{l}V \\ k\end{array}\right)$. Among these components we single out $U_{0}$, the component containing $S$. It is the only component whose closure in $\operatorname{sp}_{k} V$ does not meet $\boldsymbol{\Delta}_{k} V$. For the remaining components we can use an idea as in the proof of 7.6, and find

$$
G(V) \simeq \prod_{j \neq 0} \Gamma\left(p ; U_{j}\right)
$$

Therefore $F(V) \simeq E(V) \times G(V)$ and the composition

$$
E(V) \rightarrow F(V) \rightarrow \Gamma\left(p ; U_{0}\right) \rightarrow p^{-1}(S)
$$

is a homotopy equivalence.

Digression Knowing all the fibers of a fibration is not the same as knowing the fibration. However, in the present case we can also "describe" the entire fibration $p$ in 8.3 in terms of the cofunctor $E$. Recall from the proof of 3.5 the poset $\mathcal{I}^{(k)}$. Its elements are the open subsets of $M$ which are diffeomorphic to a disjoint union of $k$ copies of $\mathbb{R}^{m}$, and for $V, W \in \mathcal{I}^{(k)}$ we decree $V \leq W$ if and only if $V \subset W$ and the inclusion is an isotopy equivalence. We saw that

$$
\left|\mathcal{I}^{(k)}\right| \simeq\left(\begin{array}{c}
M \\
k
\end{array}\right) .
$$

Since $\mathcal{I}^{(k)} \subset \mathcal{O}$, we can restrict $E$ to $\mathcal{I}^{(k)}$. The restricted cofunctor takes all morphisms to homotopy equivalences, so that the projection

$$
\underset{\mathcal{I}^{(k)}}{\operatorname{hocolim}} E \longrightarrow\left|\mathcal{I}^{(k)}\right|
$$

is a quasifibration. The associated fibration is the one we are looking for. This motivates the following classification theorem for homogeneous cofunctors. 
8.5 Theorem Up to equivalence, all objects in $\mathcal{F}$ which are homogeneous of degree $k$ are of the type discussed in 8.3 .

Outline of proof Of course, the digression just above already gives the idea of the proof, but we have to proceed a little more cautiously. The plan is: Given $E$, homogeneous of degree but not necessarily defined in terms of some fibration, construct the appropriate $F$, polynomial of degree $\leq k$, and a morphism $E \rightarrow$ $F$. Then show that $F$ is equivalent to a cofunctor of type $V \mapsto \Gamma\left(p ;\left(\begin{array}{l}V \\ k\end{array}\right)\right)$. as in 7.1. This step requires a lemma, 8.6 below. Finally identify $E$ with the homotopy fiber of the canonical morphism from $F$ to $T_{k-1} F$.

8.6 Lemma $[3,3.12]$ Suppose that $Y$ is a functor from a small category $\mathcal{A}$ to the category of Spaces. If $Y$ takes all morphisms in $\mathcal{A}$ to homotopy equivalences, then the canonical projection $\operatorname{hocolim}_{\mathcal{A}} Y \rightarrow|\mathcal{A}|$ is a quasifibration. The section Space of the associated fibration is homotopy equivalent to $\operatorname{holim}_{\mathcal{A}} X$.

Sketch proof of 8.6 The quasifibration statement is obvious. We denote the total Space of the associated fibration by $T$, so that $\operatorname{hocolim}_{\mathcal{A}} Y \subset T$ by a homotopy equivalence. For the statement about the section Space, recall that holim $Y$ can be defined as the Space of natural transformations $\tilde{*}_{\mathcal{A}} \rightarrow Y$, where $*_{\mathcal{A}}$ is the constant functor $a \mapsto *$ on $\mathcal{A}$, and $\tilde{*}_{\mathcal{A}}$ is a $C W$-functor weakly equivalent to it (some explanations below). The standard choice is

$$
\tilde{*}_{\mathcal{A}}(a):=|\mathcal{A} \downarrow a| \text {. }
$$

$C W$-functor refers to a functor with a $C W$-decomposition where the cells are of the form $\mathbb{R}^{i} \times \operatorname{mor}(b,-)$ for some $b \in \mathcal{A}$ and some $i$. Weakly equivalent to $*_{\mathcal{A}}$ means here that there is an augmentation $\tilde{*}_{\mathcal{A}}(a) \rightarrow *_{\mathcal{A}}(a)$, natural in $a$, which is a homotopy equivalence for each $a$. In other words, $\tilde{*}_{\mathcal{A}}(a)$ is always contractible.- Suppose now that $X$ is any $\mathrm{CW}$-functor from $\mathcal{A}$ to spaces. There are obvious embeddings

$$
\operatorname{nat}(X, Y) \stackrel{\subset}{\longrightarrow} \operatorname{map}_{|\mathcal{A}|}(\operatorname{hocolim} X, \operatorname{hocolim} Y) \stackrel{\subset}{\longrightarrow} \operatorname{map}_{|\mathcal{A}|}(\operatorname{hocolim} X, T)
$$

where $\operatorname{map}_{|\mathcal{A}|}$ is for Spaces of maps over $|\mathcal{A}|$. One shows by induction over the skeletons of $X$ that the composite embedding is a homotopy equivalence. In particular, this holds for $X=\tilde{*}_{\mathcal{A}}$.

Proof of 8.5 Suppose that $E$ in $\mathcal{F}$ is homogeneous of degree $k$. Define a cofunctor $F_{0}$ from $\mathcal{O}$ to Spaces by

$$
F_{0}(V):=\underset{U \in \mathcal{I}^{(k)}(V)}{\operatorname{holim}} E(U) .
$$


Here $\mathcal{I}^{(k)}(V) \subset \mathcal{I}^{(k)}$ is the full sub-poset consisting of all $U \in \mathcal{I}^{(k)}$ which are contained in $V$. For the meaning of $\mathcal{I}^{(k)}$, see the digression preceding 8.5. By 8.6, the cofunctor $F_{0}$ is equivalent to another cofunctor $F_{1}$ given by a formula of type

$$
F_{1}(V)=\Gamma\left(q_{V}\right)
$$

where $q_{V}$ is a certain fibration on $\left|\mathcal{I}^{(k)}(V)\right|$. The fibration $q_{V}$ is natural in $W$, in the sense that a morphism $V \subset W$ in $\mathcal{O}$ induces a map from the total Space of $q_{V}$ to that of $q_{W}$, covering the inclusion

$$
\left|\mathcal{I}^{(k)}(V)\right| \hookrightarrow\left|\mathcal{I}^{(k)}(W)\right| .
$$

By inspection, this map of total Spaces maps each fiber of $q_{V}$ to the corresponding fiber of $q_{W}$ by a homotopy equivalence. Hence $F_{1}$ is equivalent to the cofunctor $F_{2}$ given by

$$
F_{2}(V):=\Gamma\left(q_{M} ;\left|\mathcal{I}^{(k)}(V)\right|\right) .
$$

Finally we know from 3.5 (and proof) that $\left|\mathcal{I}^{(k)}(V)\right| \simeq\left(\begin{array}{l}V \\ k\end{array}\right)$, and this can be understood as a chain of natural homotopy equivalences (natural in $V \in \mathcal{O}$ ). It follows easily that $F_{2}$ is equivalent to a cofunctor $F_{3}$ given by a formula of type

$$
F_{3}(V):=\Gamma\left(p ;\left(\begin{array}{l}
V \\
k
\end{array}\right)\right)
$$

where $p$ is a fibration on $\left(\begin{array}{c}M \\ k\end{array}\right)$. This is exactly the kind of cofunctor introduced in section 7 , so we now write $F:=F_{3}$. From the definition, $F$ belongs to $\mathcal{F}$. Replacing $E$ by an equivalent cofunctor if necessary, we can assume that $E$ maps directly to $F$ instead of $F_{0}$. If $S \in\left(\begin{array}{c}M \\ k\end{array}\right)$ and $V$ is a tubular neighborhood of $S \subset M$, then the composition

$$
E(V) \longrightarrow F(V)=\Gamma\left(p ;\left(\begin{array}{l}
V \\
k
\end{array}\right)\right) \stackrel{\text { eval. }}{\longrightarrow} p^{-1}(S)
$$

is a homotopy equivalence, by construction and inspection. This is of course reminiscent of 8.4. Now form the commutative square

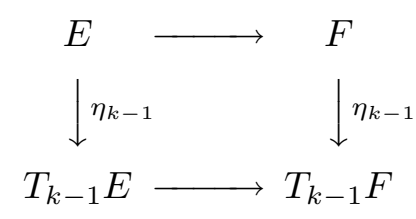

and recall that $T_{k-1} E(V)$ is contractible for all $V \in \mathcal{O}$. Given our analysis of $T_{k-1} F$ in section 7 , we can complete the proof of 8.5 by showing that $(*)$ is 
homotopy Cartesian. By 2.5 and 5.1, it suffices to check that

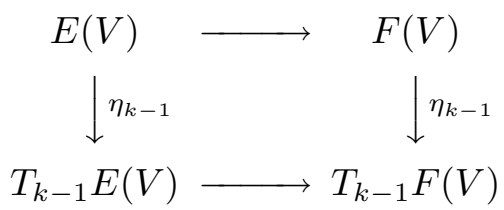

is homotopy Cartesian for all $V \in \mathcal{O} k$. If it happens that $V \in \mathcal{O} r \subset \mathcal{O} k$ for some $r<k$, then we have $E(V) \simeq *$ by homogeneity and $F(V) \rightarrow T_{k-1} F(V)$ is a homotopy equivalence, by section 5 and section 6 . If not, then $V$ has $k$ connected components and is a tubular neighborhood of some $S \subset M$, where $S \in\left(\begin{array}{c}M \\ k\end{array}\right)$. Using 8.4 now (and 7.6), and our observation above which seemed so reminiscent of 8.4 , we find that $(* *)$ is again homotopy Cartesian.

\section{The Homogeneous Layers of a Good Cofunctor}

In this section we work with a fixed $F$ in $\mathcal{F}$ and a distinguished element $* \in$ $F(M)$, which we call the base point. Since $M$ is the terminal object in $\mathcal{O}$, we may then regard $F$ as a cofunctor from $\mathcal{O}$ to pointed Spaces. Define $L_{k} F$ as in 8.2, and call it the $k$-th homogeneous layer of $F$. According to 8.5, the homogeneous cofunctor $L_{k} F$ can be classified by some fibration $p: Z \rightarrow\left(\begin{array}{c}M \\ k\end{array}\right)$, and a partial section of it defined near the fat diagonal $\boldsymbol{\Delta}_{k} M$. What does $p$ look like? The answer is implicit in the last section. Recall that

$$
\left(\begin{array}{c}
M \\
k
\end{array}\right) \simeq \mid \mathcal{I}^{(k)}
$$

in the notation of 3.5 and sequel. For any $V \in \mathcal{I}^{(k)}$ with components $V_{s}$, where $s \in \pi_{0}(V)$, the rule taking a subset $R$ of $\pi_{0}(V)$ to the Space $F\left(\cup_{s \in R} V_{s}\right)$ is a $k$-cube of Spaces:

$$
R \mapsto F\left(\cup_{s \in R} V_{s}\right) \quad\left(R \subset \pi_{0}(V)\right) .
$$

As such it has a total homotopy fiber (see 2.1) which we denote by $\Phi(V)$. Note that $V \mapsto \Phi(V)$ is a cofunctor from $\mathcal{I}^{(k)}$ to Spaces taking all morphisms to homotopy equivalences.

9.1 Proposition The fibration which classifies $L_{k} F$ is the one associated with the quasifibration

$$
\underset{V \in \mathcal{I}^{(k)}}{\operatorname{hocolim}} \Phi(V) \longrightarrow\left|\mathcal{I}^{(k)}\right| .
$$


Remark Our classifying fibrations on $\left(\begin{array}{c}M \\ k\end{array}\right)$ should always come with partial sections defined near the fat diagonal. Note that $\Phi$ is a cofunctor from $\mathcal{I}^{(k)}$ to pointed Spaces, so that the (quasi)-fibration in 9.1 does in fact have a preferred global section.

Proof of 9.1 Write $j=k-1$ (for typographic reasons). By section 8, it is enough to show that $L_{k} F(V) \simeq \Phi(V)$ for $V \in \mathcal{I}^{(k)}$, by a chain of natural pointed homotopy equivalences. Since $V \in \mathcal{I}^{(k)} \subset \mathcal{O} k$, we have

$$
\eta_{k}: F(V) \stackrel{\simeq}{\longrightarrow} T_{k} F(V)
$$

so that $L_{k} F(V)$ is homotopy equivalent to the homotopy fiber of the map $\eta_{j}: F(V) \longrightarrow T_{j} F(V)$. Recall that $T_{j} F(V)$ is defined as

$$
\operatorname{holim}_{U \in \mathcal{O}(V)} F(U) \text {. }
$$

Now observe that the inclusion of posets

$$
\left\{\cup_{s \in R} V_{s} \mid R \subset \pi_{0}(V), R \neq \pi_{0}(V)\right\} \quad \hookrightarrow \quad \mathcal{O} j(V)
$$

is right cofinal. Complete the proof by applying the cofinality theorem for homotopy inverse limits.

In the case of an embedding cofunctor, $F(V)=\operatorname{emb}(V, N)$ as in 1.3, proposition 9.1 can be made much more explicit. We need a base point in $\operatorname{emb}(M, N)$, so we may as well assume that $M$ is a smooth submanifold of $N$. For $S \in\left(\begin{array}{c}M \\ k\end{array}\right)$ let $\Psi(S)$ be the total homotopy fiber of the $k$-cube of pointed Spaces

$$
\{\operatorname{emb}(R, N) \mid R \subset S\} .
$$

These Spaces are pointed because $R \subset S \subset M \subset N$.

9.2 Theorem For $k \geq 2$, the homogeneous cofunctor $L_{k} \mathrm{emb}(-, N)$ is classified by the fibration $p: Z \rightarrow\left(\begin{array}{c}M \\ k\end{array}\right)$ with fibers $p^{-1}(S)=\Psi(S)$.

Proof The first and most important observation here is that, for every $V$ in $\mathcal{I}^{(k)}$ and every $S \in\left(\begin{array}{c}M \\ k\end{array}\right)$ which has exactly one point in each component of $V$, the obvious restriction map

$$
\rho: \Phi(V) \longrightarrow \Psi(S)
$$

is a homotopy equivalence. This can be seen as follows. For each $R \subset S$, there is a homotopy pullback square

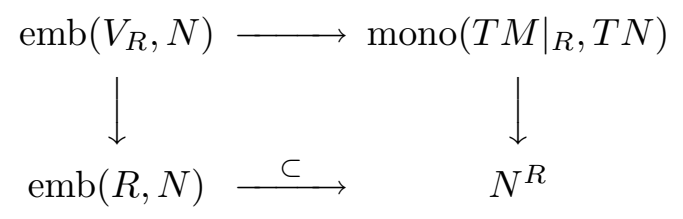

Geometry and Topology, Volume 3 (1999) 
where mono denotes a Space of bundle monomorphisms (vector bundle morphisms which are mono in each fiber of the domain). Allowing $R$ to be a variable subset of $S$ we may think of it as a square in which each vertex is a $k$-cube of Spaces. The total homotopy fibers of these $k$-cubes will then again form a homotopy pullback square. But the two $k$-cubes in the right-hand column are fibrant, so their total homotopy fibers agree with their total fibers, which reduce to a single point if $k \geq 2$ (but not if $k=1$ ). Therefore the total homotopy fibers of the $k$-cubes in the left-hand column are homotopy equivalent, which amounts to saying that $\rho: \Phi(V) \rightarrow \Psi(V)$ is a homotopy equivalence.

Now let $E_{\mathrm{b}}$ be the homotopy colimit of the cofunctor taking $V \in \mathcal{I}^{(k)}$ to the space of sections of $V \rightarrow \pi_{0}(V)$. There are obvious forgetful maps

$$
\left|\mathcal{I}^{(k)}\right| \leftarrow E_{b} \rightarrow\left(\begin{array}{c}
M \\
k
\end{array}\right) .
$$

The first of these is a homotopy equivalence by inspection. Comparison with the space $E$ in the proof of 3.5 (towards the end) shows that the second map is also a homotopy equivalence. In more detail, there is a commutative diagram

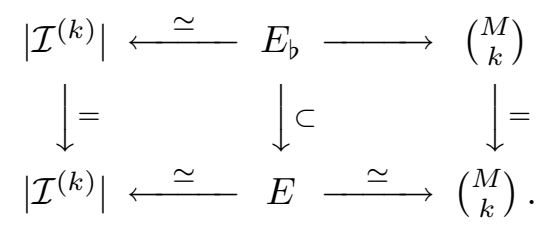

Let $p_{1}$ be the pullback of the quasifibration in 9.1 to $E_{b}$, and let $p_{2}$ be the pullback of the fibration in 9.2 to $E_{b}$. From the observation made at the beginning of this proof, it is clear that there is a map over $E_{b}$ from $p_{1}$ to $p_{2}$ which maps each fiber of $p_{1}$ to the corresponding fiber of $p_{2}$ by a homotopy equivalence.

In proposition 9.2 , the case $k=1$ has been excluded because it is different. However, it is also well understood: We have

$$
T_{1} \operatorname{emb}(-, N)=L_{1} \operatorname{emb}(-, N) \simeq \operatorname{imm}(-, N) .
$$

This follows easily from 5.1 and the observation that all arrows in the commutative square

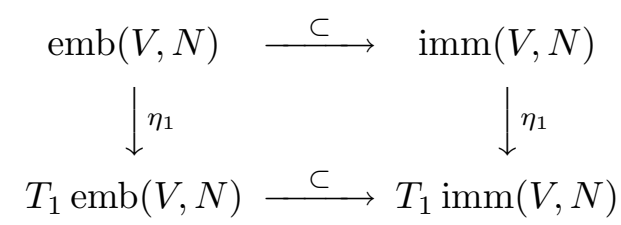

are homotopy equivalences if $V \in \mathcal{O}_{1}$. 


\section{Boundary Conditions}

So far all manifolds considered were without boundary. When there are boundaries, the theory looks slightly different. The following is an outline.

Suppose that $M^{m}$ is smooth, possibly with boundary. Let $\mathcal{O}$ be the poset of all open subsets of $M$ which contain $\partial M$. A cofunctor from $\mathcal{O}$ to Spaces is good if it satisfies conditions (a) and (b) just before 1.2, literally. In (a) we use a definition of isotopy equivalence which is appropriate for manifolds with boundary: a smooth codimension embedding $(V, \partial V) \rightarrow(W, \partial W)$ is an isotopy equivalence if, and so on.

10.1 Example Suppose that $M$ is a neat smooth submanifold of another smooth manifold $N$ with boundary. That is, $M$ meets $\partial N$ transversely, and $\partial M=M \cap \partial N$. For $V$ in $\mathcal{O}$ let $F(V)$ be the Space of smooth embeddings $V \rightarrow N$ which agree with the inclusion near $\partial M \subset V$. Then $F$ is good.

10.2 Example Suppose that $M$ is a smooth submanifold with boundary of another smooth manifold $N$ without boundary. For $V$ in $\mathcal{O}$ let $F(V)$ be the Space of smooth embeddings $V \rightarrow N$ which agree with the inclusion near $\partial M \subset V$. Then $F$ is good.

In practice example 10.1 is more important because it cannot be reduced to simpler cases, whereas 10.2 can often be so reduced. For example, with $F$ as in 10.2 there is a fibration sequence up to homotopy

$$
F(M) \rightarrow \operatorname{emb}(M \backslash \partial M, N) \rightarrow \operatorname{emb}(\partial M, N)
$$

provided $\partial M$ is compact. This follows from the isotopy extension theorem. It is a mistake to think that a similar reduction is possible in the case of 10.1. (Unfortunately I made that mistake in [23, section 5], trying to avoid further definitions; the calculations done there are nevertheless correct.)

In both examples, 10.1 and 10.2, the values $F(V)$ are contractible for collar neighborhoods $V$ of $\partial M$. For general $F$, this may not be the case.

The definition of a polynomial cofunctor of some degree $\leq k$ is again literally the same as before (2.2); we must insist that the closed subsets $A_{0}, \ldots A_{k}$ of $V \in \mathcal{O}$ have empty intersection with $\partial M$, since otherwise $F\left(V \backslash \cup_{i \in S} A_{i}\right)$ is not defined.

The definition of the full subcategory $\mathcal{O} k$ is more complicated. An element $V \in \mathcal{O}$ belongs to $\mathcal{O} k$ if it is a union of two disjoint open subsets $V_{1}$ and 
$V_{2}$, where $V_{1}$ is a collar about $\partial M$ (diffeomorphic to $\partial M \times[0,1)$ ) and $V_{2}$ is diffeomorphic to a disjoint union of $\leq k$ copies of $\mathbb{R}^{m}$.

Later we will need a certain subcategory $\mathcal{I}^{(k)}$ of $\mathcal{O} k$. An object of $\mathcal{O} k$ belongs to $\mathcal{I}^{(k)}$ if it has exactly $k$ components not meeting $\partial M$; the morphisms in $\mathcal{I}^{(k)}$ are the inclusions which are isotopy equivalences.

As before, $T_{k} F$ can be defined as the homotopy right Kan extension along $\mathcal{O} k \rightarrow \mathcal{O}$ of $F \mid \mathcal{O} k$. It turns out to be polynomial of degree $\leq k$, and it turns out that $\eta_{k}: F \rightarrow T_{k} F$ has the properties listed in 6.1.

If $F(M)$ comes with a selected base point, then we can define $L_{k} F(V)$ as the homotopy fiber of $T_{k} F(V) \rightarrow T_{k-1} F(V)$. The cofunctor $L_{k} F$ is homogeneous of degree $k$ (definition like 8.1).

A general procedure for making homogeneous cofunctors of degree $k$ on $\mathcal{O}$ is as follows. Notation: $\iota$ is the "delete boundary" command. Let $p: Z \rightarrow\left(\begin{array}{c}\iota M \\ k\end{array}\right)$ be a fibration. Suppose that it has a distinguished partial section defined near $K$, where $K$ consists of all the points in the symmetric product $\operatorname{sp}_{k} M$ having at least two identical coordinates, or having at least one coordinate in $\partial M$. For $V$ in $\mathcal{O}$ let $E(V)$ be the Space of (partial) sections of $p$ defined over $\left(\begin{array}{c}\iota V \\ k\end{array}\right)$ which agree with the distinguished (zero) section near $K$. Then $E$ is homogeneous of degree $k$.

There is a classification theorem for homogeneous cofunctors of degree $k$ on $\mathcal{O}$, to the effect that up to equivalence they can all be obtained in the way just described. The classifying fibration $p$ for a homogeneous $E$ of degree $k$ can be found/recovered as follows. Suppose that $S \subset \iota M$ has $k$ elements. Choose $V \in \mathcal{O} k$ so that $V$ contains $S \cup \partial M$ as a deformation retract. For $R \subset S$, let $V_{R}$ be the union of the components of $V$ which meet $\partial M \cup R$. Let $\Phi(V)$ be the total homotopy fiber of the $k$-cube

$$
R \mapsto F\left(V_{R}\right) .
$$

Then $\Phi(V) \simeq p^{-1}(S)$. If more detailed information is needed, one has to resort to quasifibrations: the rule $V \mapsto \Phi(V)$ can be regarded as a cofunctor on $\mathcal{I}^{(k)}$ and it gives rise to a quasifibration on $\left|\mathcal{I}^{(k)}\right| \simeq\left(\begin{array}{c}\iota M \\ k\end{array}\right)$. The associated fibration is $p$.

10.3 Example In the situation of 10.1, the classifying fibration $p_{k}$ for $L_{k} F$ has $p_{k}^{-1}(S)$ equal to the total homotopy fiber of the $k$-cube

$$
R \mapsto \operatorname{emb}(R, \iota N)
$$

for $R \subset S$, provided $k \geq 2$. The case $k=0$ is uninteresting (fiber contractible, base a single point). The case $k=1$ is different as usual; for $s \in \iota M$, the 
fiber $p_{1}^{-1}(\{s\})$ is the space of linear monomorphisms $T_{s} M \rightarrow T(\iota N)$. All this is exactly as in 9.2. For example, suppose that $M$ is compact (with boundary). Then $L_{k} F(M)$ is homotopy equivalent to the space of sections of $p_{k}$ with compact support. In other words, we are dealing with sections defined on all of the configuration space $\left(\begin{array}{c}\iota M \\ k\end{array}\right)$ and equal to the zero section outside a compact set.

\section{Acknowledgments}

Tom Goodwillie has exerted a very strong influence on this work - not only by kindly communicating his ideas of long ago to me, but also by suggesting the right analogies at the right time. Guowu Meng patiently discussed his calculations of certain embedding Spaces with me. Finally I am greatly indebted to John Klein for drawing my attention to Meng's thesis and Goodwillie's disjunction theory, on which it is based.

The author is partially supported by the NSF.

\section{References}

[1] A K Bousfield, D M Kan, Homotopy limits, completions and localizations, Lect. Notes in Math. vol. 304, Springer (1973)

[2] A Dold, Lectures on Algebraic Topology, Grundlehren series, Springer (1972)

[3] W Dwyer, The centralizer decomposition of BG, from: "Algebraic topology: new trends in localization and periodicity (San Feliu de Guixols, 1994)", Progr. in Math. 136 Birkhäuser (1996) 167-184

[4] W Dwyer, D M Kan, A classification theorem for diagrams of simplicial sets, Topology, 23 (1984) 139-155

[5] W Dwyer, M Weiss, B Williams, A parametrized index theorem for the algebraic K-theory Euler class, Parts I + II, preprint (1998)

[6] T Goodwillie, Calculus I: The first derivative of pseudoisotopy theory, KTheory, 4 (1990) 1-27

[7] T Goodwillie, Calculus II: Analytic Functors, K-Theory, 5 (1992) 295-332

[8] T Goodwillie, Calculus III: The Taylor series of a homotopy functor, in preparation

[9] T Goodwillie, A multiple disjunction lemma for smooth concordance embeddings, Memoirs Amer. Math. Soc. 86 no. 431 (1990)

[10] T Goodwillie, Excision estimates for spaces of homotopy equivalences, preprint, Brown University (1995)

[11] T Goodwillie, Excision estimates for spaces of diffeomorphisms, preprint, Brown University (1998) 
[12] T Goodwillie, J Klein, Excision estimates for spaces of Poincaré embeddings, in preparation

[13] T Goodwillie, M Weiss, Embeddings from the point of view of immersion theory: Part II, Geometry and Topology, 3 (1999) 103-118

[14] M L Gromov, Stable mappings of foliations into manifolds, Izv. Akad. Nauk. SSSR Mat. 33 (1969) 707-734, Trans. Math. USSR (Izvestia) 3 (1969) 671-693

[15] A Haefliger, V Poenaru, Classification des immersions combinatoires, Publ. Math. I.H.E.S. 23 (1964) 75-91

[16] R Kirby, L Siebenmann, Foundational Essays on Topological Manifolds, Smoothings, and Triangulations, Ann. of Math. Studies vol. 88, Princeton Univ. Press (1977)

[17] S Mac Lane, Categories for the Working Mathematician, Springer (1971)

[18] S Mac Lane, I Moerdijk, Sheaves in Geometry and Logic, Springer-Verlag (1992)

[19] V Poenaru, Homotopy and differentiable singularities, from: "Manifolds-Amsterdam 1970", Springer Lect. Notes in Math. vol. 197, 106-132

[20] G Segal, Classifying spaces related to foliations, Topology, 13 (1974) 293-312

[21] R Thomason, Homotopy colimits in the category of small categories, Math. Proc. Camb. Phil. Soc. 85 (1979) 91-109

[22] F Waldhausen, Algebraic K-theory of Spaces, from: "Proc. of 1983 Rutgers Conf. on Algebraic Topology", Lect. Notes in Math. 1126 (1985) 318-419

[23] M Weiss, Calculus of Embeddings, Bull. Amer. Math. Soc. 33 (1996) 177-187

[24] M Weiss, Curvature and finite domination, Proc. Amer. Math. Soc. 124 (1996) 615-622

[25] M Weiss, Orthogonal Calculus, Trans. Amer. Math. Soc. 347 (1995) 37433796, Erratum, Trans. Amer. Math. Soc. 350 (1998) 851-855 